\title{
Codimension-two border collision bifurcation in a two-class growth model with optimal saving and switch in behavior
}

\author{
Iryna Sushko • Pasquale Commendatore • \\ Ingrid Kubin
}

Received: 31 January 2020 / Accepted: 23 June 2020 / Published online: 18 July 2020

(C) The Author(s) 2020

\begin{abstract}
We consider a two-class growth model with optimal saving and switch in behavior. The dynamics of this model is described by a two-dimensional (2D) discontinuous map. We obtain stability conditions of the border and interior fixed points (known as Solow and Pasinetti equilibria, respectively) and investigate bifurcation structures observed in the parameter space of this map, associated with its attracting cycles and chaotic attractors. In particular, we show that on the $x$-axis, which is invariant, the map is reduced to a $1 D$ piecewise increasing discontinuous map, and prove the existence of a corresponding period adding bifurcation structure issuing from a codimension-two border collision bifurcation point. Then, we describe how this structure evolves when the related attracting cycles on the $x$-axis lose their transverse stability via a transcrit-
\end{abstract}

I. Sushko

Institute of Mathematics, NASU, 3 Tereshchenkivska St.,

Kyiv, Ukraine

e-mail: sushko@imath.kiev.ua

I. Sushko

Kyiv School of Economics, 92-94 Dmytrivska St., Kyiv, Ukraine

P. Commendatore

Department of Law, University of Naples 'Federico II',

Via Mezzocannone 16, 80134 Naples, Italy

e-mail: commenda@unina.it

I. Kubin $(\varangle)$

Vienna University of Economics and Business

Administration, Welthandelsplatz 1 Building D4,

1020 Vienna, Austria

e-mail: ingrid.kubin@wu.ac.at ical bifurcation and the corresponding interior cycles appear. In particular, we show that the observed bifurcation structure, being associated with the 2D discontinuous map, is characterized by multistability, that is impossible in the case of a standard period adding bifurcation structure.

Keywords Two-class growth model $\cdot$ Piecewise smooth discontinuous map - Border collision bifurcation $\cdot$ Period adding bifurcation structure

\section{Introduction}

The nature of long-run economic development is one of the core questions in economics. Do economies converge to a smooth growth path? Do they follow a divergent path? Are they subject to cyclical fluctuations? To irregular cycles? To sudden regime switches? Economic models addressing these questions usually view economic growth as the result of the interplay between properties of the technology and savings behavior of economic agents. While the former refers to whether productive factors are substitutes for each other and to what extent they can be substituted for each other, the latter opens the door for considering heterogeneous behavior and heterogeneous agents.

It has a long tradition differentiating between the behavior of social classes, in particular between workers and capitalists (with the latter having a higher savings propensity); in the literature on economic growth, 
this sharp distinction is often softened, as workers may also own capital (and receive a corresponding profit income). Workers' saving behavior typically differs between the different sources of income, with the savings rate out of profit income often considered to be higher than the one out of wage income (but not as high as capitalists' saving propensity). Thus, workers savings behavior can be seen as being influenced by capitalists' savings behavior in as far as profit income is concerned. In the following paper, we further develop this idea and assume that workers choose to mimic capitalists' savings behavior, once workers' capital has surpassed a threshold value. From an analytic point of view, this switching introduces a discontinuity into the model, which is otherwise two-dimensional (2D) smooth and in discrete time.

Before developing the model, we give a brief literature review, where we put emphasis on technology and savings behavior. We also cover the analytic structures of the models as well as the dynamic properties. For that, we focus on the fixed point, which is usually expressed in terms of capital per worker.

As starting point, we choose the famous HarrodDomar dilemma (see $[20,28]$ ) according to which the equilibrium growth path (which corresponds to a stationary value of capital per worker) is locally unstable. This model does not differentiate between social classes; it assumes a constant and unique saving propensity; and a technology with fixed coefficients.

During the late fifties and early sixties, two types of one-sector exogenous growth models have been proposed in the literature in order to solve the HarrodDomar dilemma. ${ }^{1}$ The Solow model [44] introduces a standard neoclassical concave production function with flexible coefficients (productive factors are substitutable with each other); this assumption leads to local and global stability of the equilibrium growth path (of the stationary value of capital per worker). Instead, Kaldor [30] introduced an early form of heterogeneous behavior by differentiating two saving propensities, attached to wages and profits, respectively. As for the previous models, the resulting dynamics is onedimensional (1D) — with the overall capital representing the only state variable - where stability is obtained

\footnotetext{
${ }^{1}$ We should mention a third category of one-sector growth models not directly spurred by the Harrod-Domar dilemma, which are the optimal growth models inspired by Ramsey [41] where the saving choices of economic agents follow an intertemporal utility maximization plan (see, for example, [2,19]).
}

by allowing variations in income distribution between different income sources adjusting saving to investment.

Pasinetti [39] reformulates Kaldor's model by introducing heterogeneous agents. In particular, he introduced two different types of agents belonging to different social classes, workers and capitalists, with differentiated saving propensities. In the Pasinetti model, the dynamics is $2 \mathrm{D}$, where workers' and capitalists' capitals represent the two state variables. An important result following from his analysis is that in a two-class stationary equilibrium-dubbed Pasinetti equilibrium - the income distribution between wages and profits - and, thus, the steady growth solution for the overall capital-is independent of workers' propensity to save ${ }^{2}$ workers saving behavior only affects the capital distribution between classes. In a later contribution, Samuelson and Modigliani [43] integrated the Solow and the Pasinetti model. Moreover, they showed that a two-class model with a flexible production technology admits another solution, other than the Pasinetti equilibrium, in which capitalists' capital is zero. In this equilibrium that replicates the properties of a Solow equilibrium, distinct groups of agents cannot be distinguished and the long-run stationary equilibrium of capital—coinciding with workers' capitalis not affected by the capitalists' propensity to save. ${ }^{3}$ These analyses were mainly concerned with the stability properties of stationary equilibria.

Quite recently, other contributions show that onesector growth models, which combine differentiated saving propensities and a technology characterized by weak factor substitutability and which are framed in discrete time, are able to generate complex dynamics, from cycles of any period to chaos. Böhm and Kass [6] introduce two saving propensities for wage and profit income respectively, as in the Kaldor model, and assume a concave production function with generic properties (it satisfies the weak Inada conditions).

\footnotetext{
2 Another striking result is that the rate of return on capital does not depend on the propensity to save of workers and on the shape of the production function. Thus, Pasinetti contribution [39] represents a critique to the marginalists approach, according to which this rate follows directly from the properties of the production function.

${ }^{3}$ It is determined by the exogenous rate of growth of population and by workers' propensity to save. In a "Solow" or "dual" equilibrium the rate of return on capital is determined by the technology, that is, it depends on the properties of the production function.
} 
These authors show that, when the two propensities to save are sufficiently different (with the propensity to save out of profits larger than the one out of wages) and factor elasticity of substitution is low, discrete-time dynamics may involve cycles of any period and chaos. The works [7-12] and [25,26] extended the Böhm and Kass model to the case of endogenous labor supply and concave or nonconcave production functions confirming the role of low factor substitutability in generating complex dynamics. Their models are 2D. However, they are confined to the study of income distribution between wage and profit income.

Commendatore [14], instead, studies a discrete-time version of the Pasinetti-Solow model put forward by Samuelson and Modigliani [43] that assumes a production function with constant elasticity of substitution (CES) and that involves two different types of agents, workers and capitalist: the ensuing model entails distributive processes also between social classes and a large variety of dynamic behaviors emerge from this formulation. In this contribution, it is shown that the distinction between a Pasinetti growth regimewhere both classes own capital—and a Solow growth regime — where only workers own capital—holds also for long-run attractors that are not stationary states. As in Solow, the propensities to save assumed by these models are fixed parameters and are not the outcome of optimizing consumer behavior.

Later contributions interested in chaotic or, more generally, complex behavior in discrete-time models assume that workers and capitalists save on the basis of rational choices. Particularly, Commendatore and Palmisani [15], in an overlapping generations setup, describe these choices on the basis of a classical approach (see [5,22] and [34]) according to which saving behavior of individuals is highly influenced by the social group (or class) to which they belong. In this framework, capitalists behave like an infinitely lived "dynasty" saving on the basis of an altruistic motive, whereas workers live only two periods and their saving pattern is driven by a life-cycle motive. The resulting model is 2D; Commendatore and Palmisani [15] present a detailed local stability analysis revealing bifurcation scenarios, which are a precondition of chaotic behavior. In [1] it is also considered an overlapping generations structure with two groups, however without the heterogeneity introduced in [15]: the two groups behave similarly, they both live two periods, and each old generation altruistically leaves a bequest to the young one. The resulting model is $2 \mathrm{D}$ and in [1] the authors explore both local and global stability properties, showing different local bifurcations and a variety of global dynamic features including multistability, complex behavior and chaos.

The present paper, which follows the previously reviewed studies in assuming a flexible production technology allowing for factor substitution, extends the previous contributions in the heterogeneous behavior dimension. Following [15], we consider two social classes, workers and capitalists that behave differently; following [1], altruism and the bequest motive may play a role not only for capitalists', but also for workers' savings decision. Several contributions in the literature suggest that there is a link between wealth accumulation and the bequest motive (see [17] and [16]). In our framework, for capitalists this link is always present: capitalists behave altruistically and leave bequests, thus providing for their "dynasty." Instead, workers have two behavioral patterns: when their wealth is low, their saving pattern is driven by the usual life-cycle motive; instead, when their wealth crosses a certain threshold, workers saving behavior switches to the more altruistic, bequest leaving behavior imitating the capitalists' social class behavior.

Our analysis not only confirms the main results of the literature-according to which 2D models with differential saving propensities (and thus distribution processes between income types and social classes) and weak factor substitutability may lead to chaotic behavior. With the introduction of a switch in workers saving choices, the resulting model is discontinuous and is also able to show a larger set of complex dynamics features.

In fact, modeling a switch in the behavior of workers leads to a discontinuous dynamical system, namely to a 2D piecewise smooth (PWS) map $F$, corresponding to the full system describing the dynamic evolution of workers' and capitalists' capital, with one discontinuity line, called switching manifold. Maps of this class appear also in other applications (see, e.g., $[13,24,27,42,46])$ and belong to a relatively new research field which is not yet well developed. In particular, in the bifurcation theory of PWS maps there are open problems related to border collision bifurcations ${ }^{4}$ (BCBs) and to the corresponding bifurcation structures

\footnotetext{
$\overline{4}$ A border collision bifurcation occurs when an invariant set of a PWS map collides with its switching manifold under variation of some parameter, and such a collision leads to a qualitative
} 
observed in the parameter space of such maps. In this respect, continuous PWS maps have the advantage that, to classify possible outcomes of a $\mathrm{BCB}$, one can use border collision normal forms, represented by continuous piecewise linear (PWL) maps, which are quite well studied (see, e.g., $[18,37,38,45])$. Some results related to dynamics of discontinuous PWL maps can be found in $[21,35,40]$.

Among discontinuous PWS maps the most studied are 1D piecewise monotone maps with one discontinuity point, associated with Lorenz like flows (to cite a few, see [3,29,31-33], etc.). It is known that investigation of bifurcation structures observed in the parameter space of such maps often leads to specific parameter points associated with intersection of different BCB curves (see, e.g., [23] and references therein). For example, in case of a 1D discontinuous piecewise increasing map, an intersection point of two curves related to BCBs of two different fixed points or cycles can be the origin of a period adding bifurcation structure, associated with attracting cycles, or of a bandcount adding bifurcation structure, related to chaotic attractors. If a $1 \mathrm{D}$ discontinuous map with increasing and decreasing branches is considered, the related intersection point can give rise to period/bandcount incrementing bifurcation structures. In [4] these structures are described in detail in case of a 1D discontinuous PWL map; and examples of 1D PWS maps with similar and mixed bifurcation structures are also presented.

It is interesting to investigate how the mentioned above structures evolve when a 2D discontinuous map is considered. In fact, in the parameter space of map $F$, we observe a set which is an intersection of $\mathrm{BCB}$ boundaries of the stability regions of different fixed points, and from this set a bifurcation structure issues which has some similarities with a period adding structure. We show that on the $x$-axis (which is invariant) map $F$ is reduced to a $1 \mathrm{D}$ piecewise increasing map $g-$ which describes the dynamic evolution of the system when only workers own capital-for which we prove the existence of a period adding bifurcation structure associated with attracting cycles belonging to the $x$ axis. Then, we investigate how this structure is modified when the related attracting cycles lose their transverse

Footnote 4 continued

change in the dynamics. This notion is introduced in [37] where 2D continuous PWS maps are considered. stability via transcritical bifurcation and corresponding interior cycles appear. The main peculiarity of the observed structure is related to multistability, which is impossible in the case of a standard period adding structure. For example, in the standard period adding structure, between 2- and 3-periodicity regions there is a 5 -periodicity region, ${ }^{5}$ and all these regions are disjoint, while in the observed structure such regions can overlap, leading to coexistence of 2-, 3- and 5-cycles.

The paper is organized as follows. In Sect. 2, we put forward the general economic setup, describing the saving behavior of the two groups and deriving the accumulation law for capitalists' and workers' capital. In Sect. 3, we analyze the dynamics of map $F$ depending on its parameters, namely, in Sect. 3.1, we obtain stability conditions of the interior fixed points; in Sect. 3.2, we present several examples of the bifurcation structures observed in the parameter space of map $F$ and associated with its attracting cycles and chaotic attractors; in Sect. 3.3, we analyze the dynamics of the 1D map $g$ and, in particular, we obtain the stability conditions of the border fixed points and prove the existence of a period adding structure issuing from a codimension-two BCB point, associated with the border fixed points; in Sect. 3.4, we show how this structure evolves when attracting cycles, related to this period adding structure, lose their transverse stability. In Sect. 4, we propose some conclusions.

\section{General setup of the model}

The analysis is framed in discrete time and we denote by $t$ the time unit (or period). We consider a one-good economy, where production only involves two factors, capital $K_{t}$ and labor $L_{t}$. A constant fraction $0 \leq \delta \leq 1$ of capital depreciates in each period. The production technology is of the constant elasticity of substitution (CES) type and takes the following functional form:

$f\left(k_{t}\right)= \begin{cases}\left(1-\alpha+\alpha k_{t}^{\rho}\right)^{1 / \rho} & \text { if } \rho \leq 1, \rho \neq 0 \\ k_{t}^{\alpha} & \text { if } \rho=0\end{cases}$

where $k_{t}=K_{t} / L_{t}$ is the capital/labor ratio, $0<\alpha<1$ is the distribution coefficient, $-\infty<\rho<1(\rho \neq 0)$ the substitution coefficient and $(1-\rho)^{-1}$ the elasticity

\footnotetext{
5 Recall that a period adding structure is formed by the periodicity regions related to attracting cycles, and these regions are ordered according to the Farey summation rule applied to the rotation numbers of the related cycles (see, e.g., [4] for details).
} 
of substitution. Notice that when the coefficient related to the elasticity of factor substitution tends to zero, i.e., if $\rho \rightarrow 0$, we have the case of a Cobb-Douglas production function-i.e., intermediate factor substitutability and flexible production factor coefficients; instead, we have the case of a Leontief technology-i.e., no substitutability and fixed production factor coefficients-if $\rho \rightarrow-\infty$; and the case of perfect substitutability if $\rho=1$.

Capital is the only asset in the economy, and wages and profits are the only sources of income. We define a short-run equilibrium as a situation in which perfectly competitive capital and labor markets ensure equality between the profit rate $r_{t}$ and the marginal product of capital $f^{\prime}\left(k_{t}\right)$, and between the wage rate $w_{t}$ and the marginal product of labor $f\left(k_{t}\right)-f^{\prime}\left(k_{t}\right) k$. For the case of a CES technology, this implies:

$$
\begin{aligned}
r_{t} & =f^{\prime}\left(k_{t}\right) \\
& = \begin{cases}\alpha\left[(1-\alpha) k_{t}^{-\rho}+\alpha\right]^{1 / \rho-1} & \text { if } \rho \leq 1, \rho \neq 0 \\
\alpha k_{t}^{\alpha-1} & \text { if } \rho=0\end{cases} \\
w_{t} & =f\left(k_{t}\right)-f^{\prime}\left(k_{t}\right) k \\
& = \begin{cases}(1-\alpha)\left[(1-\alpha) k_{t}^{-\rho}+\alpha\right]^{1 / \rho-1} k_{t}^{1-\rho} & \text { if } \rho \leq 1, \rho \neq 0 \\
(1-\alpha) k_{t}^{\alpha-1} & \text { if } \rho=0\end{cases}
\end{aligned}
$$

The economy is inhabited by two types of agents grouped into social classes, workers (denoted by the subscript $w$ ) and capitalists (denoted by the subscript $c$ ). Both classes live two periods according to an overlapping generations structure, have the same population size, $L_{t}$, and grow at the same rate, $n \geq 0$. Moreover, both workers and capitalists are endowed with logarithmic preferences. Capitalists earn only income out of capital, we denote $K_{c, t}$ the capital of this type of agents. They consume only a fraction of their wealth when old and leave what is left to the current young generation (their offspring). A young capitalist only decides how much to consume out of her wealth in her first period of life and saves what is left for the next period. Each worker supplies inelastically one unit of labor when young and does not work when old. If workers' capital is small, they consume all their wealth when old and do not leave any bequest to the young generation. If workers' capital is sufficiently high, they behave like capitalists. They consume only a fraction of their wealth and leave what is left to the current young generation. We denote $K_{w, t}$ the capital of this type of agents.

In order to determine capitalists and workers savings and accumulation choices, we proceed as follows. Starting from the old generation of period $t$, each old capitalist consumes only a fraction $\left(1-d_{c}\right)$ of her wealth, and pays to her descendants the fraction $d_{c}$. A young capitalist faces an intertemporal utility maximization problem, following the assumption of logarithmic preferences, the solution involves saving a fixed proportion of her wealth $s_{c, t}=\beta_{c} d_{c}\left(1-\delta+r_{t}\right) k_{c, t}$, where $s_{c, t}$ is the saving of a young capitalist and $\beta_{c}$ is capitalists' consumption time discount factor. Considering workers, again we start from the old generation of period $t$ : if her capital is smaller than a certain threshold, $k_{w, t}<\bar{k}$, an old worker consumes all her wealth (income plus depreciated capital), where $k_{w, t}$ denotes workers' capital expressed in terms of labor units. She doesn't leave anything to the young generation of period $t$. When $k_{w, t}<\bar{k}$, in period $t$, young workers do not receive any transfer from the old generation. The solution of the intertemporal utility maximization problem gives a saving corresponding to $s_{w, t}=\beta_{w 1} w_{t}$, where $s_{w, t}$ is the saving of a young worker in period $t$ and $\beta_{w 1}$ is workers' consumption time discount factor when their capital is low. Instead, if her capital is larger than that threshold, $k_{w, t}>\bar{k}$, an old worker consumes only a fraction $\left(1-d_{w}\right)$ of her wealth and pays to her descendants the fraction $d_{w}$. When $k_{w, t}>\bar{k}$, in period $t$, the solution of a worker intertemporal utility maximization problem gives a saving corresponding to $s_{w, t}=\beta_{w 2}\left[w_{t}+d_{w}\left(1-\delta+r_{t}\right) k_{w, t}\right]$, where $\beta_{w 2}$ is workers' consumption time discount factor when their capital is high.

In such a way, the main dynamic equations governing capitalists and workers accumulation differ depending on the size of workers' capital, namely, capital accumulation is determined by the following system of difference equations, or, in other words, by a family of $2 \mathrm{D}$ piecewise smooth discontinuous maps:

$$
\begin{cases}k_{c, t+1}=\frac{1}{1+n} s_{c, t}=\frac{1}{1+n} \beta_{c} d_{c}\left(1-\delta+r_{t}\right) k_{c, t} & \text { if } k_{w, t}<\bar{k} \\ k_{w, t+1}=\frac{1}{1+n} s_{w, t}= \begin{cases}\frac{1}{1+n} \beta_{w 1} w_{t} & \text { if } k_{w, t}>\bar{k} \\ \frac{1}{1+n} \beta_{w 2}\left[w_{t}+d_{w}\left(1-\delta+r_{t}\right) k_{w, t}\right.\end{cases} \end{cases}
$$


where the following inequalities are satisfied: $0<$ $\beta_{w 1}, \beta_{w 2}, \beta_{c}<1, \bar{k}>0$, and $k_{t}=k_{c, t}+k_{w, t}$.

\section{Bifurcation structure of the parameter space}

In this section, we explore the dynamic properties of the system (3). What is useful to notice from an economic point of view is that this system is able to generate two different growth regimes: a Pasinetti regime, in which both capitalists and workers own capital, and a Solow regime, in which only workers own a positive amount of capital. The dynamics corresponding to each regime may undergo a strong qualitative change depending on the saving behavior of workers. This behavior is modified when the threshold $\bar{k}$ is crossed. and

$$
D_{L}=\{(x, y): x<\bar{x}\}, \quad D_{R}=\{(x, y): x>\bar{x}\}
$$

The discontinuity line which separates the $(x, y)$-phase plane into the halfplanes $D_{L}$ and $D_{R}$, is denoted $D L$ :

$D L=\{(x, y): x=\bar{x}\}$

One can notice immediately that the $x$-axis is an $F$ invariant line, on which map $F$ is reduced to a 1D PWS discontinuous map

$$
\begin{aligned}
g & : x \rightarrow g(x) \\
& = \begin{cases}g_{L}(x)=w_{1}\left(f(x)-x f^{\prime}(x)\right) & \text { if } x<\bar{x} \\
g_{R}(x)=w_{2}(x+f(x)) & \text { if } x>\bar{x}\end{cases}
\end{aligned}
$$

Considering the CES production function $f(k)$ given in (1), and assuming that $\rho<1, \rho \neq 0$, two maps defining map $F$ can be written in the following form:

$$
\begin{aligned}
& F_{L}:\left(\begin{array}{l}
x \\
y
\end{array}\right) \rightarrow\left(\begin{array}{l}
w_{1}(1-\alpha)\left(1-\alpha+\alpha(x+y)^{\rho}\right)^{1 / \rho-1} \\
c y\left(1+\alpha\left(\alpha+(1-\alpha)(x+y)^{-\rho}\right)^{1 / \rho-1}\right)
\end{array}\right) \\
& F_{R}:\left(\begin{array}{l}
x \\
y
\end{array}\right) \rightarrow\left(\begin{array}{l}
w_{2}\left(x+\left(1-\alpha+\alpha(x+y)^{\rho}\right)^{1 / \rho-1}\left(1-\alpha+\alpha x(x+y)^{\rho-1}\right)\right) \\
c y\left(1+\alpha\left(\alpha+(1-\alpha)(x+y)^{-\rho}\right)^{1 / \rho-1}\right)
\end{array}\right)
\end{aligned}
$$

For the dynamic analysis, we impose the following values for the parameters: $n=0$ and $d_{c}=d_{w}=1$. The last assumption corresponds to the case in which old generations do not consume. Moreover, we set $\delta=0$. This will simplify the mathematical analysis reducing the number of parameters. Let us also introduce the following notations for the variables and parameters:

$k_{w}=x, k_{c}=y, \beta_{c}=c, \beta_{w 1}=w_{1}, \beta_{w 2}=w_{2}, \bar{k}=\bar{x}$

Putting everything together, taking into account the short-run equilibrium conditions and dropping the time subscript, the family of $2 \mathrm{D}$ discontinuous piecewise smooth maps defined in (3) can be written as follows:

$F:(x, y) \rightarrow\left\{\begin{array}{l}F_{L}(x, y) \text { if }(x, y) \in D_{L} \\ F_{R}(x, y) \text { if }(x, y) \in D_{R}\end{array}\right.$

where

$$
\begin{aligned}
& F_{L}:\left(\begin{array}{l}
x \\
y
\end{array}\right) \rightarrow\left(\begin{array}{l}
w_{1}\left(f(x+y)-(x+y) f^{\prime}(x+y)\right) \\
c y\left(1+f^{\prime}(x+y)\right)
\end{array}\right) \\
& F_{R}:\left(\begin{array}{l}
x \\
y
\end{array}\right) \rightarrow\left(\begin{array}{l}
w_{2}\left(x+f(x+y)-y f^{\prime}(x+y)\right) \\
c y\left(1+f^{\prime}(x+y)\right)
\end{array}\right)
\end{aligned}
$$

A 1D map $g$, to which $F$ is reduced on the $x$-axis, is defined as

$$
\begin{aligned}
g & : x \rightarrow g(x) \\
& = \begin{cases}g_{L}(x)=w_{1}(1-\alpha)\left(1-\alpha+\alpha x^{\rho}\right)^{1 / \rho-1} & \text { if } x<\bar{x} \\
g_{R}(x)=w_{2}\left(x+\left(1-\alpha+\alpha x^{\rho}\right)^{1 / \rho}\right) & \text { if } x>\bar{x}\end{cases}
\end{aligned}
$$

The dynamics of map $g$ is described in Sect. 3.3. Note that it does not depend on the parameter $c$, representing capitalists' propensity to save.

Let us collect all the parameter conditions to be satisfied:

$0<c, w_{1}, w_{2}, \alpha<1, \rho<1, \rho \neq 0, \bar{x}>0$

\subsection{Interior fixed points and their bifurcations}

The long-run stationary equilibria of the economic model correspond to the fixed points of map $F$. In this section, where we focus especially on interior equilibria, and in Sect. 3.3, we identify these fixed points and explore their local stability properties. Also for the fixed points we are able to distinguish between long-term positions where a Pasinetti regime prevails - these are 
called Pasinetti equilibria and correspond to the interior fixed points of map $F$ - and where a Solow regime prevails - these are called Solow equilibria and correspond to border equilibria of map $F$. There are two types of Pasinetti and Solow equilibria, depending on their location with respect to the threshold $x=\bar{x}$.

To get a fixed point of map $F$, first note that the function defining the dynamics of the $y$-variable is the same for both maps, $F_{L}$ and $F_{R}$. From $y=c y\left(1+f^{\prime}(x+y)\right)$, it follows that at any fixed point of $F$, say, $(x, y)=$ $\left(x^{*}, y^{*}\right)$, with $y^{*} \neq 0$ (Pasinetti equilibrium), the following equality holds:

$f^{\prime}\left(x^{*}+y^{*}\right)=\frac{1-c}{c}$

and from (2), we have that

$x^{*}+y^{*}=\left(\frac{1-\alpha}{b-\alpha}\right)^{1 / \rho}$

where

$b:=\left(\frac{1-c}{c \alpha}\right)^{\rho /(1-\rho)}$

Notice also that

$f^{\prime \prime}\left(x^{*}+y^{*}\right)=-\frac{(1-\rho)(1-c)(b-\alpha)^{1+1 / \rho}}{b c(1-\alpha)^{1 / \rho}}$

One of the feasibility conditions for the fixed point is $x^{*}+y^{*}>0$, and a sufficient condition for this is

$b-\alpha>0$

Substituting $b$ from (13) into the above inequality, one can see that it is equivalent to

$\alpha<\left(\frac{1-c}{c}\right)^{\rho}$

Let the related boundary in the parameter space be denoted as

$B: \quad \alpha=\left(\frac{1-c}{c}\right)^{\rho}$

Note that the inequality (16) can also be written as

$c<\frac{1}{\alpha^{1 / \rho}+1}, \quad 0<\rho<1$

and

$c>\frac{1}{\alpha^{1 / \rho}+1}, \quad \rho<0$

In the parameter space of map $F$, a region satisfying (10) and (16) is called a feasible domain:

$$
\begin{aligned}
\Phi= & \left\{c, w_{1}, w_{2}, \alpha, \rho, \bar{x}: 0<c, w_{1}, w_{2}, \alpha<1, \rho<1,\right. \\
& \left.\rho \neq 0, \bar{x}>0, \alpha<(1 / c-1)^{\rho}\right\}
\end{aligned}
$$

and from now on we suppose that the parameter values belong to $\Phi$.

Consider map $F_{L}$. Let $L\left(x_{L}, y_{L}\right)$ denote a fixed point of $F_{L}$ where $y_{L} \neq 0$, i.e., $L\left(x_{L}, y_{L}\right)$ is a Pasinetti equilibrium, which is a stationary equilibrium involving positive workers' and capitalists' capital. Substituting (12) into

$x=w_{1}(1-\alpha)\left(1-\alpha+\alpha(x+y)^{\rho}\right)^{1 / \rho-1}$

we get that fixed point $L$ is defined by

$$
\left\{\begin{array}{l}
x_{L}=w_{1} \frac{(1-c)(b-\alpha)}{\alpha c}\left(\frac{1-\alpha}{b-\alpha}\right)^{1 / \rho} \\
y_{L}=\left(\frac{1-\alpha}{b-\alpha}\right)^{1 / \rho}\left(1-\frac{w_{1}(1-c)(b-\alpha)}{\alpha c}\right)
\end{array}\right.
$$

For the parameter values belonging to the parameter domain $\Phi$, it follows that $x_{L}>0$, while $y_{L}>0$ if

$w_{1}<\frac{\alpha c}{(b-\alpha)(1-c)}$

The fixed point $L$ is an actual fixed point ${ }^{6}$ of $F$, if $x_{L}<\bar{x}$, that holds for

$w_{1}<\frac{\bar{x} \alpha c(b-\alpha)^{1 / \rho-1}}{(1-c)(1-\alpha)^{1 / \rho}}$

and a BCB of $L$ occurs if $x_{L}=\bar{x}$, that is, if the parameter values satisfy the following equality:

$B C_{L}: \quad w_{1}=\frac{\bar{x} \alpha c(b-\alpha)^{1 / \rho-1}}{(1-c)(1-\alpha)^{1 / \rho}}$

Map $F_{L}$ can also have fixed point(s) belonging to the $x$-axis, corresponding to Solow equilibria, involving zero capitalists' capital. First note that, for $\rho<0$ the origin is a fixed point of $F$, denoted $O(0,0)$, but not for $\rho>0$. Let $L^{*}\left(x_{L}^{*}, 0\right)$ denote a fixed point with $x_{L}^{*} \neq 0$, where $x=x_{L}^{*}$ is a fixed point of map $g_{L}$ given in (9), that is, it satisfies the equality

$x=w_{1}(1-\alpha)\left(1-\alpha+\alpha x^{\rho}\right)^{1 / \rho-1}$

The fixed point $L^{*}\left(x_{L}^{*}, 0\right)$ is an actual fixed point of map $F$ if $x_{L}^{*}<\bar{x}$, and a BCB of $L^{*}$ occurs if $x_{L}^{*}=\bar{x}$, i.e., if

$B C_{L^{*}}: \quad w_{1}=\frac{\bar{x}}{(1-\alpha)\left(1-\alpha+\alpha \bar{x}^{\rho}\right)^{1 / \rho-1}}$

In fact, depending on the parameter values, $g_{L}$ can have zero, one or two nontrivial fixed points. The second

${ }^{6}$ Recall that an actual fixed point belongs to the region, where the corresponding map, in this case $F_{L}$, is defined. 
fixed point, if it exists, is denoted $L^{* *}\left(x_{L}^{* *}, 0\right)$, where $x_{L}^{* *}$ also satisfies (25), $x_{L}^{* *}<x_{L}^{*}$ (see Sect. 3.3 for details).

Later we show that the condition $y_{L}=0$, leading to

$T_{L}: \quad w_{1}=\frac{\alpha c}{(b-\alpha)(1-c)}$

(see (22)), is associated with a transcritical bifurcation of the fixed points $L$ and $L^{*}$ (or $L^{* *}$ ) at which these fixed points merge, and then the border fixed point changes its transverse stability (i.e., the stability in the direction which is transverse to the $x$-axis).

Now, we derive the fixed points of map $F_{R}$. Assuming $y \neq 0$ and substituting (12) into

$$
\begin{aligned}
x= & w_{2}\left(x+\left(1-\alpha+\alpha(x+y)^{\rho}\right)^{1 / \rho-1}\right. \\
& \left.\left(1-\alpha+\alpha x(x+y)^{\rho-1}\right)\right)
\end{aligned}
$$

(see (8)), we get that one fixed point of $F_{R}$, denoted $R\left(x_{R}, y_{R}\right)$, is given by

$$
\left\{\begin{array}{l}
x_{R}=\frac{w_{2}(1-c)(b-\alpha)}{\alpha\left(c-w_{2}\right)}\left(\frac{1-\alpha}{b-\alpha}\right)^{1 / \rho} \\
y_{R}=\left(\frac{1-\alpha}{b-\alpha}\right)^{1 / \rho}\left(1-\frac{w_{2}(1-c)(b-\alpha)}{\alpha\left(c-w_{2}\right)}\right)
\end{array}\right.
$$

Taking into account the parameter conditions defining the feasible domain $\Phi$ (see (20)), it can be seen that both inequalities $x_{R}>0$ and $y_{R}>0$ are satisfied if

$w_{2}<\frac{\alpha c}{b(1-c)+\alpha c}$

given that this condition together with the condition (15) implies that $c-w_{2}>0$. The fixed point $R$ is an actual fixed point of $F$ if $x_{R}>\bar{x}$, that is, if

$$
w_{2}>\frac{\bar{x} \alpha c}{(1-c)(1-\alpha)^{1 / \rho}(b-\alpha)^{1-1 / \rho}+\bar{x} \alpha}
$$

and a BCB of $R$ occurs if

$$
B C_{R}: \quad w_{2}=\frac{\bar{x} \alpha c}{(1-c)(1-\alpha)^{1 / \rho}(b-\alpha)^{1-1 / \rho}+\bar{x} \alpha}
$$

Map $F_{R}$ can also have a nontrivial fixed point belonging to the $x$-axis (see Sect. 3.3 for details). ${ }^{7}$ Let $R^{*}\left(x_{R}^{*}, 0\right)$ be such a fixed point, where $x=x_{R}^{*}, x_{R}^{*}>0$, is a fixed point of map $g_{R}$ given in (9), obtained from $x=w_{2}\left(x+\left(1-\alpha+\alpha x^{\rho}\right)^{1 / \rho}\right)$ :

$x_{R}^{*}=\left(\frac{1-\alpha}{\left(\frac{1}{w_{2}}-1\right)^{\rho}-\alpha}\right)^{1 / \rho}$

\footnotetext{
${ }^{7}$ For $\rho<0$ the origin is a fixed point of map $F_{R}$; however, for $\bar{x}>0$ it is never a fixed point of map $F$.
}

The fixed point $R^{*}\left(x_{R}^{*}, 0\right)$ is an actual fixed point of $F$ if $x_{R}^{*}>\bar{x}$, and a BCB of $R^{*}$ occurs if

$\bar{x}=w_{2}\left(\bar{x}+\left(1-\alpha+\alpha \bar{x}^{\rho}\right)^{1 / \rho}\right)$

that is, if

$B C_{R^{*}}: \quad w_{2}=\frac{\bar{x}}{\bar{x}+\left(1-\alpha+\alpha \bar{x}^{\rho}\right)^{1 / \rho}}$

Below we show that the equality $y_{R}=0$, which is satisfied if

$T_{R}: \quad w_{2}=\frac{\alpha c}{b(1-c)+\alpha c}$

(see (29)), corresponds to a transcritical bifurcation of the fixed points $R$ and $R^{*}$, at which these fixed points merge, and then $R^{*}$ changes its transverse stability.

Now we obtain the conditions for the other bifurcations of the fixed points. The Jacobian matrix of $F_{L}$ is defined as

$D F_{L}=\left(\begin{array}{ll}-w_{1} k f^{\prime \prime}(k)-w_{1} k f^{\prime \prime}(k) \\ c y f^{\prime \prime}(k) & c y f^{\prime \prime}(k)+c\left(1+f^{\prime}(k)\right)\end{array}\right)$

First, note that for $\rho<0$, the origin (which in such a case is a fixed point of $F_{L}$ ) is always a saddle in the feasible domain, with eigenvalue $\lambda_{1}=0$ in the horizontal direction and $\lambda_{2}=c\left(1+f^{\prime}(0)\right)>1$ in the transversal direction. The latter inequality simplifies to (19), so that crossing the boundary $B$ (see (17)) related to $\lambda_{2}=1$, a transcritical bifurcation occurs leading to the appearance/disappearance of the interior fixed point $L$ in the positive quadrant of the phase plane.

From (11) it follows that $c\left(1+f^{\prime}\left(k_{L}\right)\right)=1$, where $k_{L}=x_{L}+y_{L}$, with $x_{L} \neq 0, y_{L} \neq 0$, thus,

$$
\left.D F_{L}\right|_{\left(x_{L}, y_{L}\right)}=\left(\begin{array}{ll}
-w_{1} k_{L} f^{\prime \prime}\left(k_{L}\right) & -w_{1} k_{L} f^{\prime \prime}\left(k_{L}\right) \\
c y_{L} f^{\prime \prime}\left(k_{L}\right) & c y_{L} f^{\prime \prime}\left(k_{L}\right)+1
\end{array}\right)
$$

The stability of $L$ is defined by the following system of inequalities:

$\left\{\begin{array}{l}1-\left(c y_{L} f^{\prime \prime}\left(k_{L}\right)+1-w_{1} k_{L} f^{\prime \prime}\left(k_{L}\right)\right)-w_{1} k_{L} f^{\prime \prime}\left(k_{L}\right)>0 \\ 1+\left(c y_{L} f^{\prime \prime}\left(k_{L}\right)+1-w_{1} k_{L} f^{\prime \prime}\left(k_{L}\right)\right)-w_{1} k_{L} f^{\prime \prime}\left(k_{L}\right)>0 \\ -w_{1} k_{L} f^{\prime \prime}\left(k_{L}\right)<1\end{array}\right.$

The first condition in (35) is reduced to $c y_{L} f^{\prime \prime}\left(k_{L}\right)<$ 0 . This condition is satisfied if $y_{L}>0$, given that $f^{\prime \prime}\left(k_{L}\right)<0$ (see (14)). If the condition (27), associated with $y_{L}=0$, holds, then a transcritical bifurcation of $L$ occurs (of course, $L$ has to be an actual fixed point of $F$, that is, the condition (23) must also hold). 
The second condition in (35) can be written as

$w_{1}>\frac{c y_{L} f^{\prime \prime}\left(k_{L}\right)+2}{2 f^{\prime \prime}\left(k_{L}\right) k_{L}}$

It is satisfied if

$w_{1}>\frac{c \alpha((1-\rho)(b-\alpha)(1-c)-2 b)}{(1-\rho)(b-\alpha)(1-c)((1-c)(b-\alpha)+2 \alpha)}$

and a flip bifurcation of the actual fixed point $L$ occurs if

$$
\begin{aligned}
& F l_{L}: \quad w_{1} \\
& =\frac{c \alpha((1-\rho)(b-\alpha)(1-c)-2 b)}{(1-\rho)(b-\alpha)(1-c)((1-c)(b-\alpha)+2 \alpha)}
\end{aligned}
$$

The third condition in (35) holds if

$$
c b-w_{1}(1-\rho)(b-\alpha)(1-c)>0
$$

Taking into account the condition (15), the above inequality can be written as

$w_{1}<\frac{c b}{(1-\rho)(1-c)(b-\alpha)}$

and if

$N S_{L}: \quad w_{1}=\frac{c b}{(1-\rho)(1-c)(b-\alpha)}$

a Neimark-Sacker bifurcation of $L$ occurs.

The Jacobian matrix of $F_{R}$ evaluated at the fixed point $R$ is defined as

$$
\begin{aligned}
D & \left.F_{R}\right|_{\left(x_{R}, y_{R}\right)} \\
= & \left(\begin{array}{lr}
w_{2}\left(1+f^{\prime}\left(k_{R}\right)-y_{R} f^{\prime \prime}\left(k_{R}\right)\right) & -w_{2} y_{R} f^{\prime \prime}\left(k_{R}\right) \\
c y_{R} f^{\prime \prime}\left(k_{R}\right) & c y_{R} f^{\prime \prime}\left(k_{R}\right)+1
\end{array}\right) \\
= & \left(\begin{array}{lr}
w_{2}\left(1-c y_{R} f^{\prime \prime}\left(k_{R}\right)\right) / c-w_{2} y_{R} f^{\prime \prime}\left(k_{R}\right) \\
c y_{R} f^{\prime \prime}\left(k_{R}\right) & c y_{R} f^{\prime \prime}\left(k_{R}\right)+1
\end{array}\right)
\end{aligned}
$$

where $k_{R}=x_{R}+y_{R}$. The stability conditions of $R$ are given by implies that $w_{2}<c$, that is, the third condition in (39) is satisfied as well. This also means that the fixed point $R$ cannot undergo a Neimark-Sacker bifurcation, associated with the third condition.

The second condition in (39) is reduced to

$c-w_{2}<\frac{4 c}{2-c y_{R} f^{\prime \prime}\left(k_{R}\right)}$

Substituting the expressions for $f^{\prime \prime}\left(k_{R}\right)$ and $y_{R}$ (see (14) and (28), respectively) into the above inequality, we get that the second condition in (39) is satisfied if

$w_{2}>\frac{c \alpha((1-\rho)(1-c)(b-\alpha)-2 b)}{(1-\rho)(1-c)(b-\alpha)(b(1-c)+c \alpha)+2 \alpha b}$

and a flip bifurcation of an actual fixed point $R$ occurs if

$$
\begin{aligned}
& F l_{R}: \quad w_{2} \\
& =\frac{c \alpha((1-\rho)(1-c)(b-\alpha)-2 b)}{(1-\rho)(1-c)(b-\alpha)(b(1-c)+c \alpha)+2 \alpha b}
\end{aligned}
$$

To summarize, we can state the following

Proposition 1 Consider map $F$ given in (4) defined by maps $F_{L}$ and $F_{R}$, given in (7) and (8), respectively, with parameter values belonging to the feasible domain $\Phi$ defined in (20). Then, map $F$ has an actual attracting fixed point $L$ with coordinates given in (21), if the following inequalities are simultaneously satisfied:

$$
\left\{\begin{aligned}
w_{1} & <\frac{\bar{x} \alpha c(b-\alpha)^{1 / \rho-1}}{(1-c)(1-\alpha)^{1 / \rho}} \\
w_{1} & <\frac{\alpha c}{(b-\alpha)(1-c)} \\
w_{1} & >\frac{c \alpha(1-\rho)(b-\alpha)(1-c)-2 b \alpha c}{(1-\rho)(b-\alpha)(1-c)((1-c)(b-\alpha)+2 \alpha)} \\
w_{1} & <\frac{c b}{(1-\rho)(1-c)(b-\alpha)}
\end{aligned}\right.
$$

and map $F$ has an actual attracting fixed point $R$ with coordinates given in (28), if the following inequalities are simultaneously satisfied:

$$
\left\{\begin{array}{l}
1-\left(w_{2}\left(1-c y_{R} f^{\prime \prime}\left(k_{R}\right)\right) / c+c y_{R} f^{\prime \prime}\left(k_{R}\right)+1\right)+w_{2} / c>0 \\
1+w_{2}\left(1-c y_{R} f^{\prime \prime}\left(k_{R}\right)\right) / c+c y_{R} f^{\prime \prime}\left(k_{R}\right)+1+w_{2} / c>0 \\
w_{2} / c<1
\end{array}\right.
$$

The first condition in (39) is reduced to

$y_{R} f^{\prime \prime}\left(k_{R}\right)\left(w_{2}-c\right)>0$

Recall that $f^{\prime \prime}\left(k_{R}\right)<0$, while $y_{R}>0$ if the condition given in (29) is satisfied. If the condition (33), associated with $y_{R}=0$, holds, then a transcritical bifurcation of $R$ occurs. Moreover, for $b-\alpha>0$ the condition (29) $\left\{\begin{aligned} w_{2} & >\frac{\bar{x} \alpha c}{(1-c)(1-\alpha)^{1 / \rho}(b-\alpha)^{1-1 / \rho}+\bar{x} \alpha} \\ w_{2} & >\frac{c \alpha((1-\rho)(1-c)(b-a)-2 b)}{(1-\rho)(1-c)(b-a)(b(1-c)+c \alpha)+2 \alpha b} \\ w_{2} & <\frac{\alpha c}{b(1-c)+\alpha c}\end{aligned}\right.$

Proposition 1 summarizes all possible locally stable stationary equilibria that can exist in a Pasinetti regime. 


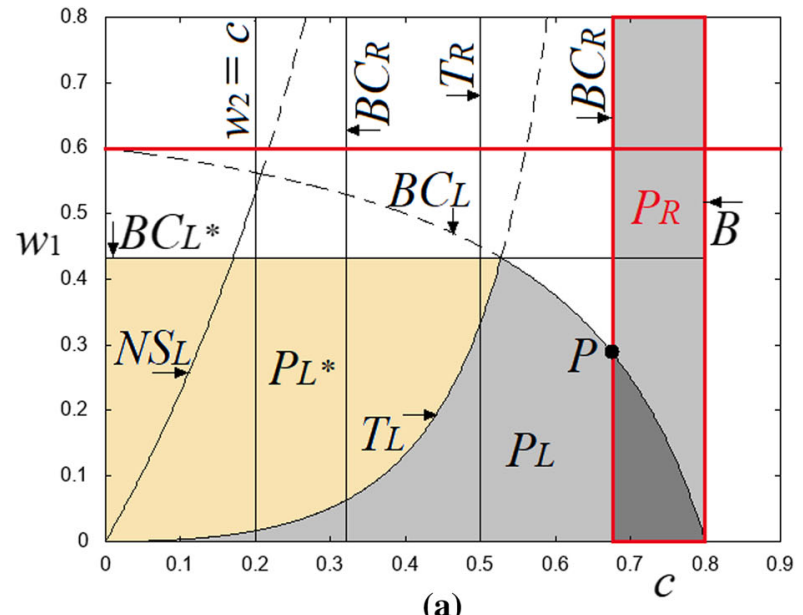

(a)

Fig. 1 Bifurcation curves confining the existence and stability regions of the fixed points of map $F$ in the $\left(c, w_{1}\right)$-parameter plane for $w_{2}=0.2$ in $(\mathbf{a})$, and in the $\left(c, w_{2}\right)$-parameter plane for

These are differentiated depending on their position with respect to the threshold $\bar{x}$.

As mentioned in the Introduction, one of our purposes is to describe the bifurcation structures associated with an intersection of the BCB boundaries of the interior attracting fixed points of map $F$. Let $P$ denote such a parameter set:

$P=B C_{L} \cap B C_{R}=\left\{\begin{array}{l}w_{1}=\frac{\bar{x} \alpha c(b-\alpha)^{1 / \rho-1}}{(1-c)(1-\alpha)^{1 / \rho}} \\ w_{2}=\frac{\bar{x} \alpha c}{(1-c)(1-\alpha)^{1 / \rho}(b-\alpha)^{1-1 / \rho}+\bar{x} \alpha}\end{array}\right.$

Given that at $P$ it holds that $x_{L}=x_{R}=\bar{x}$, leading to $w_{1}=\frac{c w_{2}}{c-w_{2}}$

this equality can also be used to define $P$, instead of one of those given in (42). It follows also that the set $P$ satisfies

$c>w_{2}$

As we discuss in Sect. 3.4, the set $P$ can serve as an organizing center from which many other bifurcation boundaries issue.

\subsection{Examples of the bifurcation}

structures in the parameter space of map $F$

In this section, we present a few examples of the stability regions of the actual fixed points of map $F$ for different parameter settings, as well as the related bifurcation

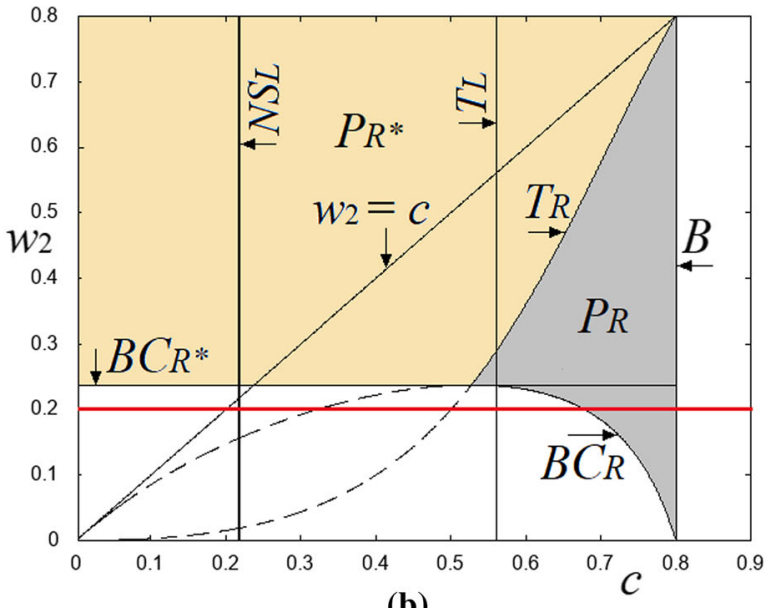

(b)

$w_{1}=0.6$ in (b). Other parameters are fixed as $\rho=0.5, \alpha=0.5$, $\bar{x}=0.15$

structures, emphasizing some interesting features associated with the discontinuity of the map. We will show how these bifurcation structures change depending on parameter values. We will also stress some aspects which are relevant from an economic point of view.

In the feasible parameter domain $\Phi$ given in (20), a region denoted $P_{L}$ and associated with an actual interior attracting fixed point $L$, can be confined by the boundaries $B C_{L}, T_{L}, F l_{L}, N S_{L}$, defined in (24), (27), (36), (38), respectively, while a region denoted $P_{R}$ and related to an actual interior attracting fixed point $R$, can be confined by the boundaries $B C_{R}, T_{R}, F l_{R}$, defined in (30), (33), (41), respectively.

Let first the parameter values be fixed as $\rho=0.5$, $\alpha=0.5, \bar{x}=0.15$. In Fig. 1a, for $w_{2}=0.2$, we show the bifurcation curves in the $\left(c, w_{1}\right)$-parameter plane, and in Fig. 1b, for $w_{1}=0.6$, in the $\left(c, w_{2}\right)$ parameter planes. The regions $P_{L}$ and $P_{R}$ are shown in light gray and their overlapping parts, related to the coexisting fixed points $L$ and $R$, are shown in dark gray; the regions $P_{L^{*}}$ and $P_{R^{*}}$, associated with attracting border fixed point $L^{*}$ and $R^{*}$, respectively, are shown in light yellow. In the considered case, the region $P_{L^{*}}$ is confined by the curves $T_{L}$ and $B C_{L^{*}}$ (see (27) and (26), respectively), and the region $P_{R^{*}}$ is bounded by the curves $T_{R}$ and $B C_{R^{*}}$ (see (33) and (32), respectively). Note that for $\rho=0.5$, the condition (16), which can be written as in (18), is satisfied for $c<0.8$, that is, the boundary $B$ (see 17) corresponds to $c=0.8$. Note also 

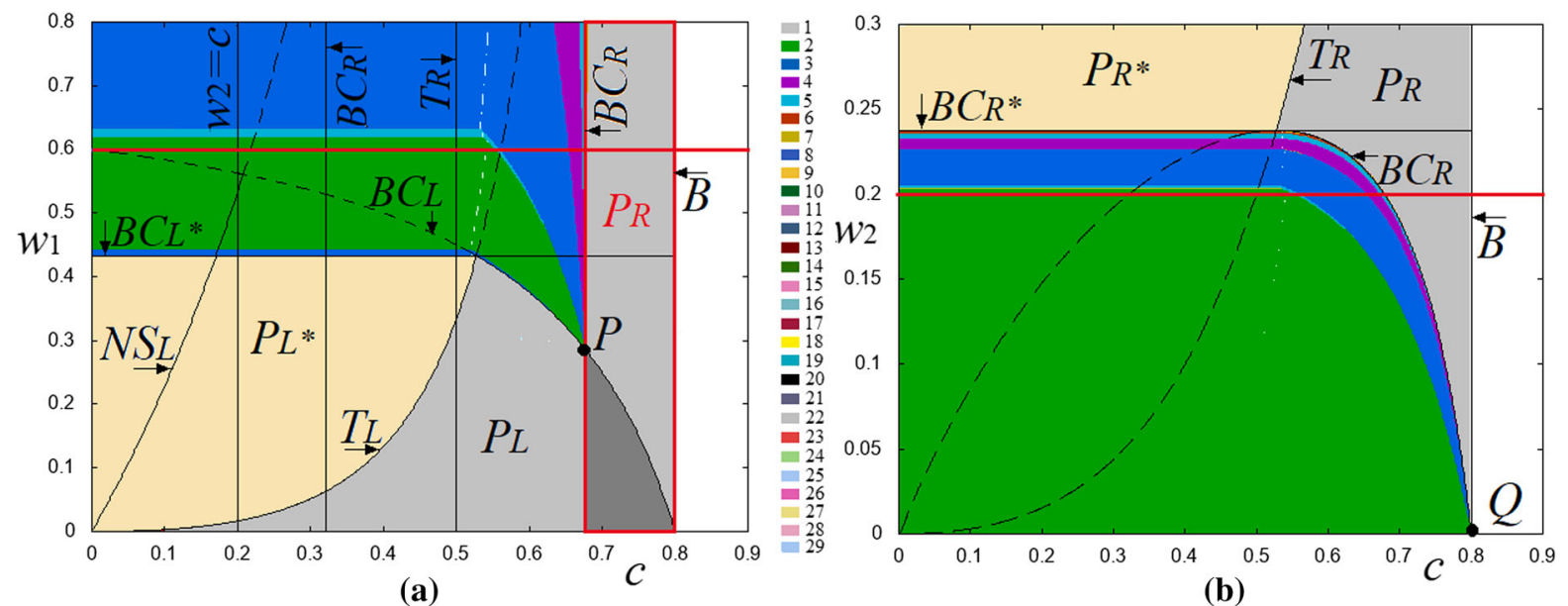

Fig. 22 bifurcation diagram of map $F$ in the $\left(c, w_{1}\right)$-parameter plane for $w_{2}=0.2$ in (a), and in the $\left(c, w_{2}\right)$-parameter plane for $w_{1}=0.6$ in (b). Other parameters are fixed as $\alpha=0.5, \rho=0.5, \bar{x}=0.15$

that if a parameter point tends to the boundary $B$, then the fixed point $R$ tends to infinity. In Fig. 1a the horizontal line defined by $w_{1}=0.6$ and related to Fig. $1 \mathrm{~b}$ is marked, and in Fig. 1b it is marked the horizontal line with $w_{2}=0.2$, associated with Fig. 1a, so that one can compare the intersection points of these horizontal lines with the bifurcation curves. One can also see how the overlapping parts of the stability regions change if $w_{2}$ varies as in Fig. 1a, or if $w_{1}$ varies as in Fig. $1 b$. For example, from Fig. 1b it is easy to see that starting from $w_{2}=0.2$, decreasing $w_{2}$, the region $P_{R}$ decreases and, thus, in Fig. 1a an overlapping part of $P_{L}$ and $P_{R}$ decreases as well. If $w_{2}$ is increasing, the region $P_{R}$ at first increases, but then, when $w_{2}$ becomes larger than the value defining the boundary $B C_{R^{*}}$, it decreases. At the same time, in Fig. 1a the region $P_{R^{*}}$ appears, overlapping with both stability regions, $P_{L}$ and $P_{L^{*}}$. It is interesting to notice here that when $\rho>0$ there is coexistence of different types of long-run stationary equilibria, in particular, $R$ may coexist with $L$, and $R^{*}$ with $L$ and $L^{*}$, whereas there is no evidence that $L^{*}$ can coexist with $R$.

Figure 2a presents the complete bifurcation structure of the $\left(c, w_{1}\right)$-parameter plane, superimposed on the related bifurcation curves shown in Fig. 1a. Here, different colors are associated with attracting cycles of different periods $n \leq 30$ (the correspondence between colors and periods is indicated in the middle panel). One can immediately see that from point $P$, defined by $\left(c, w_{1}\right) \approx(0.67838,0.28362)$ (see $\left.(42)\right)$, different periodicity regions are issuing. Figure $2 b$ shows an enlarged part of the bifurcation structure of the $\left(c, w_{2}\right)$ parameter plane together with the bifurcation curves presented in Fig. 1b, and it can be seen that in this plane the periodicity regions originate from the point $Q$ defined by $\left(c, w_{2}\right)=(0.8,0)$, which is a tangency point of the curves $B$ and $B C_{R}$. In Fig. 3a, we present a bifurcation structure of the $\left(w_{1}, w_{2}\right)$-parameter plane for $c=0.6$, where, in particular, one can notice that some periodicity regions issuing from point $P$, defined by $\left(w_{1}, w_{2}\right) \approx(0.375,0.23077)$, are overlapping with the region $P_{R}$. As an example in the phase plane, see Fig. $3 \mathrm{~b}$ where an attracting fixed point $R$ and an attracting 7-cycle are shown together with their basins for $w_{1}=0.55, w_{2}=0.231$. Note that the basins are separated by a segment of the discontinuity line $D L$ and its preimages.

It is convenient to represent an $n$-cycle $\left\{\left(x_{i}, y_{i}\right)\right\}_{i=0}^{n-1}$, $n \geq 2$, of map $F$ by its symbolic sequence $\sigma=$ $\sigma_{0} \ldots \sigma_{n-1}$, where $\sigma_{i} \in\{L, R\}$ and

$\sigma_{i}= \begin{cases}L & \text { if } x_{i}<\bar{x} \\ R & \text { if } x_{i}>\bar{x}\end{cases}$

For example, the 7-cycle shown in Fig. $3 \mathrm{~b}$ has the symbolic sequence $L R^{6}$. For short, we denote a cycle by its symbolic sequence.

The periodicity regions with horizontal boundaries, which can be seen in Fig. 2, form a period adding bifurcation structure (as we prove in Sect. 3.3), being associated with the dynamics restricted to the $x$-axis, namely, with attracting $n$-cycles, $n \geq 2$, of map $g$ given in (9). By increasing $c$ each of such $n$-cycle loses its transverse stability via a transcritical bifurcation lead- 


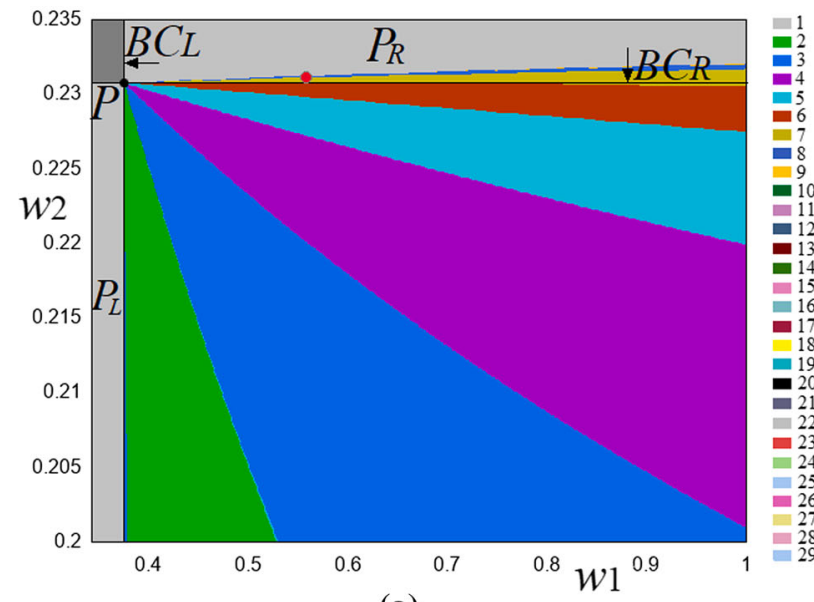

(a)

Fig. 3 a 2 D bifurcation diagram of map $F$ in the $\left(w_{1}, w_{2}\right)$ parameter plane for $c=0.6$; b coexisting attracting fixed point $R$ and an attracting 7-cycle together with their basins for

ing to the appearance of an attracting interior $n$-cycle in the positive quadrant of the phase plane (such as, for example, the 7-cycle shown in Fig. 3b). This transformation occurs in a similar way as the one, which happens to the fixed point $L^{*}$ (or $R^{*}$ ) losing its transverse stability when the curve $T_{L}$ (or $T_{R}$, respectively) is crossed and an interior attracting fixed point $L$ (or $R$, respectively) appears in the positive quadrant. So, the observed bifurcation structure can be seen as an extension of the period adding structure of the 1D discontinuous map $g$ to a specific bifurcation structure of the 2D discontinuous map $F$.

One more example is presented in Fig. 4, where $\rho=-1, \alpha=0.2, \bar{x}=0.5$ : we show in Fig. 4a the bifurcation structure of the $\left(c, w_{1}\right)$-parameter plane for $w_{2}=0.35$, and in Fig. $4 \mathrm{~b}$ the bifurcation structure of the $\left(c, w_{2}\right)$-parameter planes for $w_{1}=0.75$. For the considered parameter values the region $P_{L}$ is confined by the curves $N S_{L}, T_{L}, B C_{L}$ and $B$ (where $B$ is defined by $c=1 / 6$, see (17)); the region $P_{L^{*}}$ is bounded by the curves $T_{L}, B C_{L^{*}}$ and $F_{L^{*}}$ (the later curve corresponds to a fold bifurcation leading to the appearance of attracting and repelling fixed points, $L^{*}$ and $L^{* *}$, as explained in Sect. 3.3, see (44)); the region $P_{R}$ is bounded by the curves $T_{R}$ and $B C_{R}$; region $P_{R^{*}}$ is confined by the curves $T_{R}$ and $B C_{R^{*}}$. Similar to the previous example, the point $P$ is an issue point of other bifurcation curves; and, as we prove in Sect. 3.3, the periodicity regions

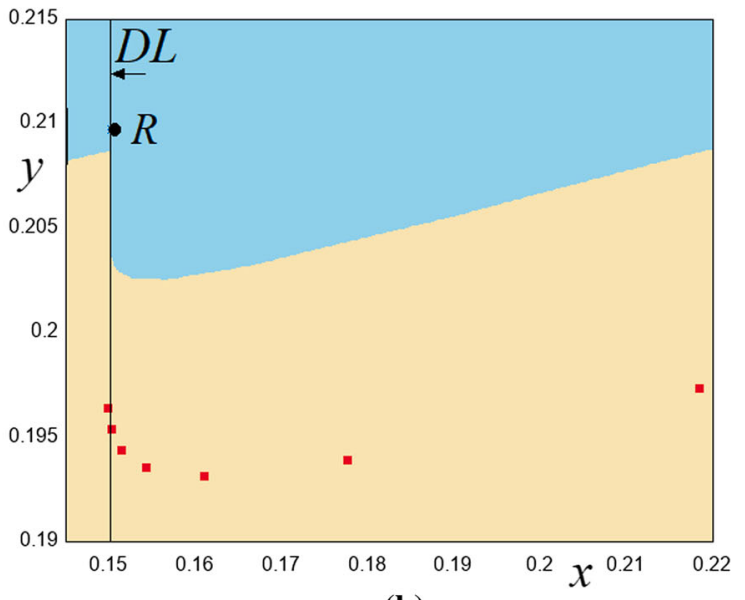

(b)

$w_{1}=0.55, w_{2}=0.231$ [the related parameter point is marked by red circle in (a)]. Other parameters are fixed as $\alpha=0.5$, $\rho=0.5, \bar{x}=0.15$. (Color figure online)

with horizontal boundaries, observed in Fig. 4a, also form a period adding structure. An overall bifurcation structure, associated with this example, is discussed in more detail in Sect. 3.4. Note that in the considered case the fixed point $L$ can undergo a Neimark-Sacker bifurcation, namely, when a parameter point crosses the boundary $N S_{L}$ of region $P_{L}$, leading to the appearance of an attracting closed invariant curve $C$. (In Fig. 4 the white regions are related to such curves.) It is also worth to note that curve $C$ can coexist with the fixed point $L^{*}$, or with the fixed point $R^{*}$, or with an attracting cycle belonging to the $x$-axis and associated with the period adding structure mentioned above. See, for example, Fig. 5 where we present the curve $C$ coexisting with a border 3 -cycle together with their basins. ${ }^{8}$ By increasing $c$, the curve $C$ disappears after a contact

\footnotetext{
${ }^{8}$ Recall that the only positive quadrant of the phase plane is feasible; however, in Fig. 5 we present the complete basin of $C$ in order to show that its boundary is formed by the stable invariant sets of the saddle fixed points $O$ and $L^{* *}$. (In Fig. $5 L_{-1}^{* *}$ denotes a preimage of $L^{* *}$.) Such a structure of the basin is possible due to the noninvertibility of map $F_{L}$ : in Fig. 5 a we show also the straight line $L C_{-1}=\{(x, y): x=-y\}$ associated with the vanishing determinant of $D F_{L}$ (recall that det $D F_{L}=-w_{1}(x+$ y) $f^{\prime \prime}(x+y)$ ); its image $F_{L}\left(L C_{-1}\right)=L C=\{(x, y): x=0\}$ is called critical line. For the theory of critical lines see [36]. It would be interesting to study the bifurcation phenomena related to the interaction of the images/preimages of the discontinuity line $D L$ and line $L C_{-1}$ for different parameter values. However, this problem is out of the scope of the present paper.
} 


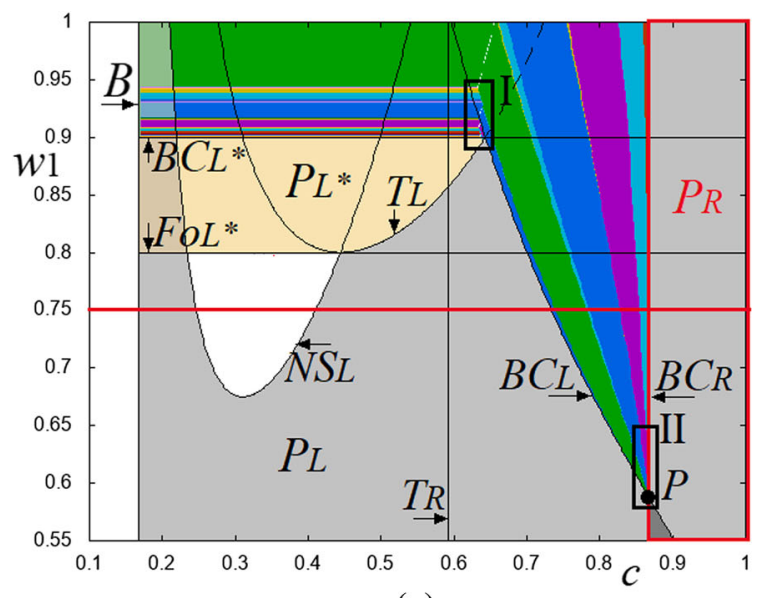

(a)

Fig. $42 \mathrm{D}$ bifurcation diagram of map $F$ in the $\left(c, w_{1}\right)$ parameter plane for $w_{2}=0.35$ in (a), and in the $\left(c, w_{2}\right)$ parameter plane for $w_{1}=0.75$ in $(\mathbf{b})$. Other parameters are

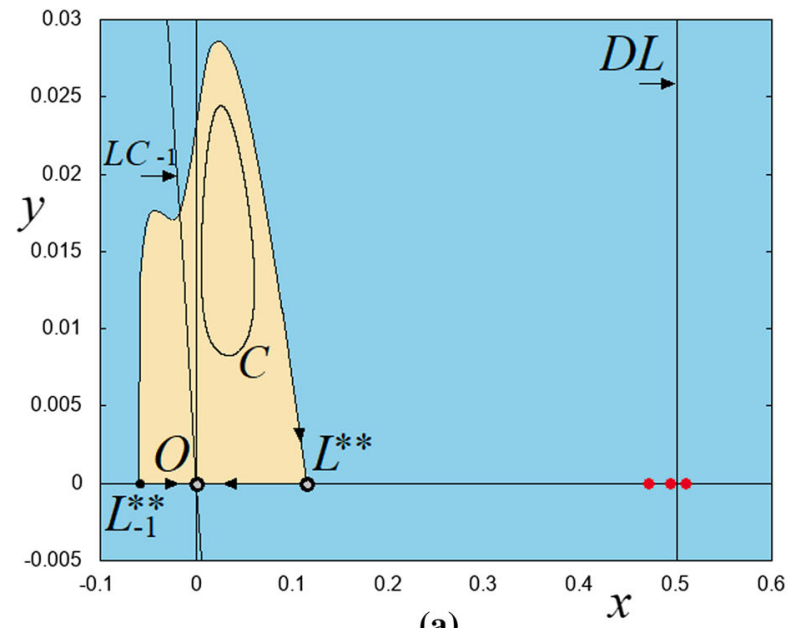

(a)

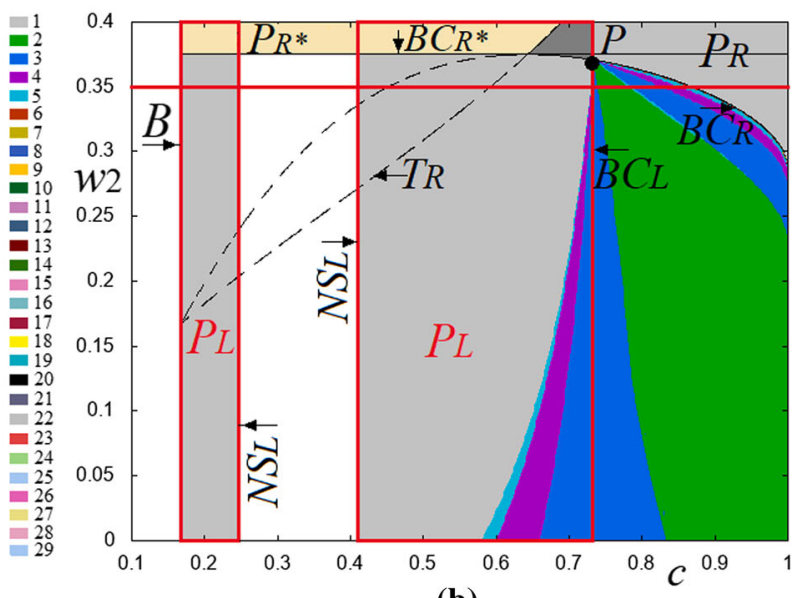

(b)

fixed as $\alpha=0.2, \rho=-1, \bar{x}=0.5$. Windows I and II, indicated in (a), are shown enlarged in Figs. 13 and 15a, respectively

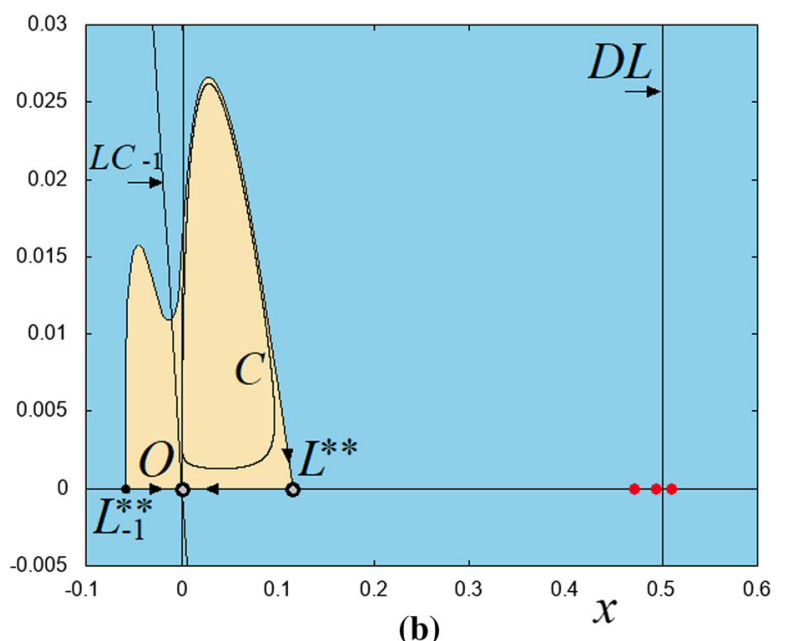

(b)

Fig. 5 Coexisting closed invariant attracting curve $C$ and an attracting border 3-cycle, together with their basins. Here $\rho=-1, \alpha=0.2$, $\bar{x}=0.5, w_{1}=0.925, w_{2}=0.35$ and $c=0.22$ in $(\mathbf{a}), c=0.224$ in (b)

with its basin boundary (a homoclinic bifurcation of the fixed point $O$ occurs): see Fig. $5 \mathrm{~b}$ where the curve $C$ is near to contact its basin boundary.

By lower values of $\rho$-i.e., for weaker factor substitutability - the dynamics of map $F$ become more complicated. Without going into details, we show in Fig. 6a the bifurcation structure of the $\left(c, w_{1}\right)$ parameter plane for $\rho=-7, \alpha=0.5, \bar{x}=0.15, w_{2}=$ 0.2 . It can be seen that the point $P$ is still an organizing center from which other bifurcation curves issue. One can notice also that periodicity regions related to cycles of different periods are overlapping with each other. Figure $6 \mathrm{~b}$ illustrates the phase plane in case of coexistence of three interior attracting cycles, $R L^{5}, R L^{6}$ and $R L^{5} R L^{6}$, and an attracting closed invariant curve $C$. Note that, in this case, the basin of $C$ is bounded by a segment of the discontinuity line $D L$ and its preimages.

By further decreasing $\rho$, the flip bifurcation curves $F l_{L}$ and $F l_{R}$, given in (36) and (41), respectively, also 'enter' the feasible parameter domain $\Phi$ : see, for exam- 


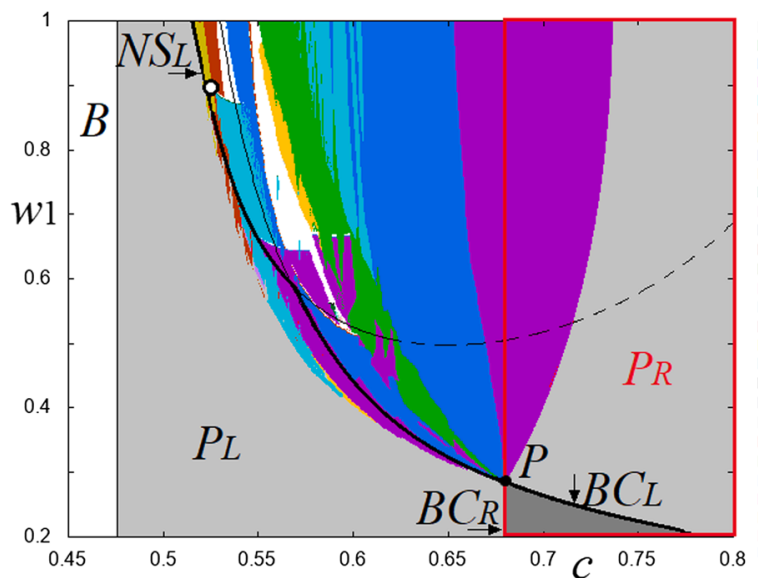

(a)

Fig. 6 a $2 \mathrm{D}$ bifurcation diagram of map $F$ in the $\left(c, w_{1}\right)$ parameter plane for $w_{2}=0.2 \alpha=0.5, \rho=-7, \bar{x}=0.15$; $\mathbf{b}$ an attracting closed invariant curve $C$ coexisting with attracting

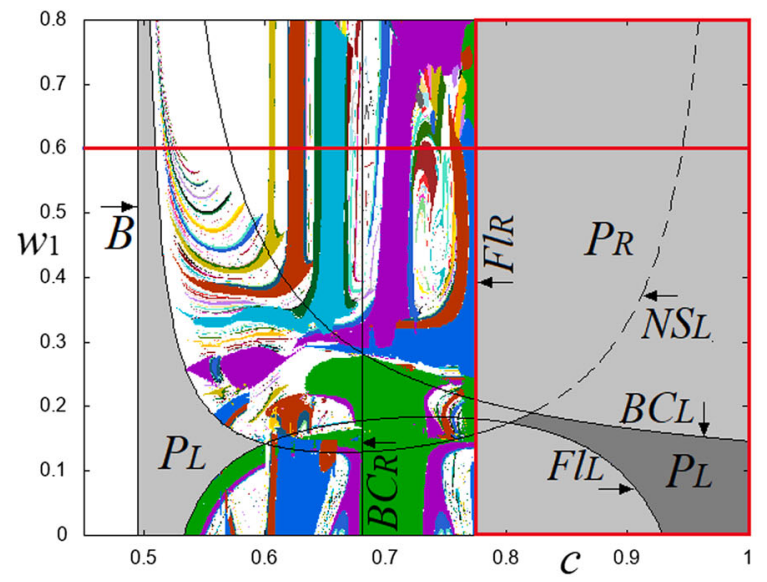

(a)
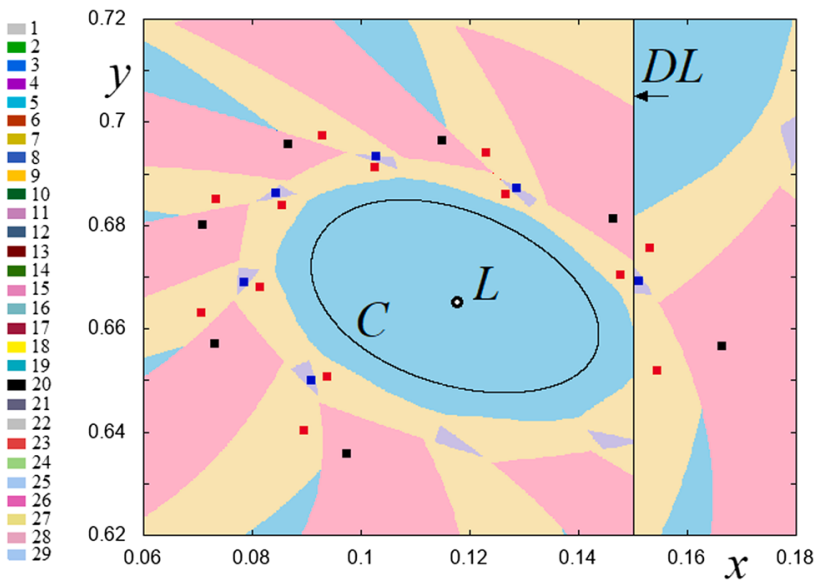

(b)

6-, 7- and 13-cycles, together with their basins, for $w_{1}=0.895$, $c=0.523$ [the related parameter point is marked by white circle in (a)]

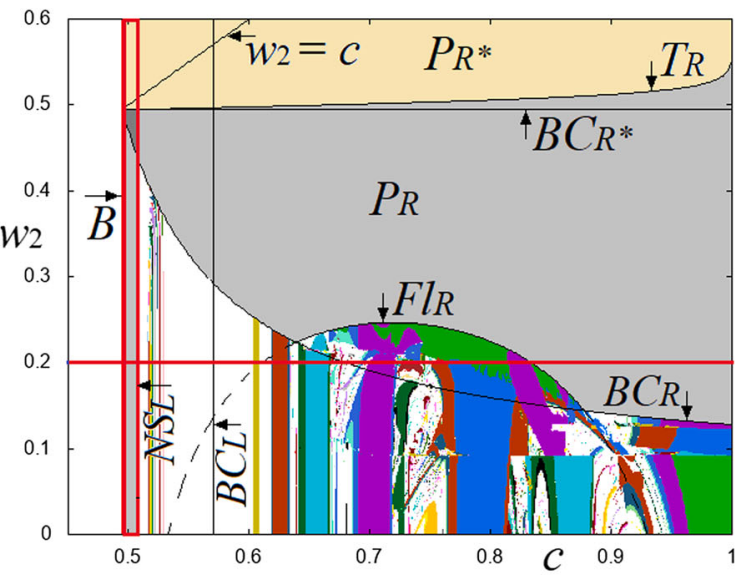

(b)

Fig. 7 Bifurcation structure in the $\left(w_{1}, c\right)$-parameter plane for $w_{2}=0.2(\mathbf{a})$ and in the $\left(w_{2}, c\right)$-parameter plane for $w_{1}=0.6(\mathbf{b})$. Other parameters are fixed as $\rho=-30, \alpha=0.5, \bar{x}=0.15$

ple, the bifurcation structures for $\rho=-30$ in Fig. 7. Namely, in Fig. 7 a the $\left(c, w_{1}\right)$-parameter plane is shown for $w_{2}=0.2$, and in Fig. $7 \mathrm{~b}$ the $\left(c, w_{2}\right)$-parameter plane is shown for $w_{1}=0.6$. Note that for the parameter setting related to Fig. $7 \mathrm{a}$, the region $P_{L}$ is split into two parts: one part is confined by the curves $B, N S_{L}$ and $F l_{L}$, while the other part (overlapped with the region $\left.P_{R}\right)$ is confined by the curves $N S_{L}, B C_{L}$ and $F l_{L}$.

To illustrate some nonsmooth bifurcations, we present in Fig. 8a 1D bifurcation diagrams $c$ versus $x$ related to a cross section of Fig. 7 a for $w_{1}=0.19$. We know that in such a case, by increasing $c$, the fixed point $L$ undergoes a Neimark-Sacker bifurcation leading to an attracting closed invariant curve; it can be seen that by further increasing $c$, a fold bifurcation opens a window associated with an attracting 3-cycle, which follows a standard sequence of bifurcations leading to chaos, and then a nonsmooth expansion bifurcation of a 6piece chaotic attractor occurs caused by its contact with the discontinuity line $D L$ (see the arrow marked (1) in Fig. 8a). One can also notice that by decreasing $c$, a 4-cycle born via a flip bifurcation of a 2-cycle, under- 


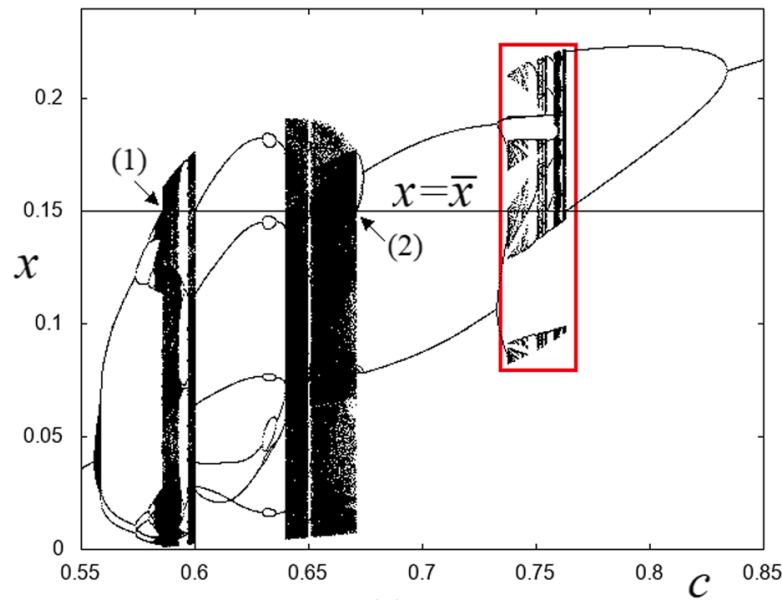

(a)

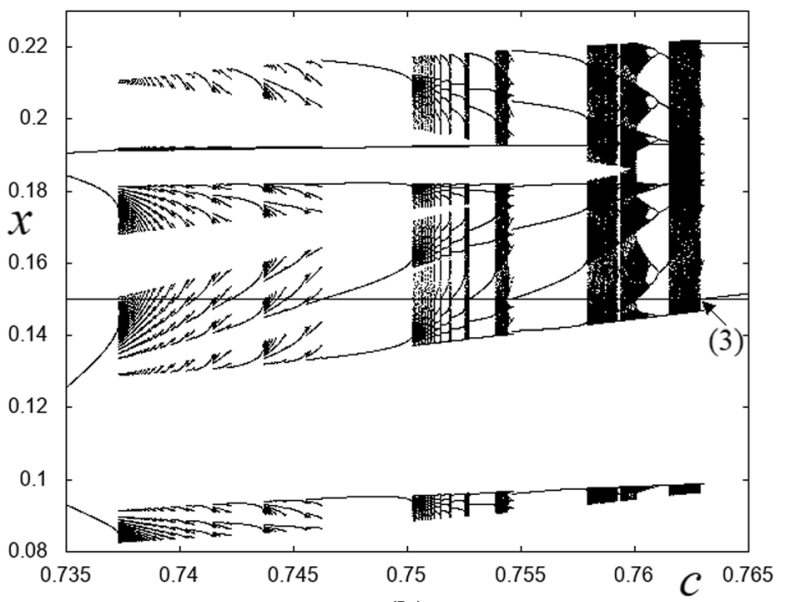

(b)

Fig. 8 a 1D bifurcation diagram $c$ versus $x$ of map $F$ for $w_{1}=0.19, w_{2}=0.2, \rho=-30, \alpha=0.5, \bar{x}=0.15$; $\mathbf{b}$ an enlargement of the window indicated in (a)

goes a BCB leading directly to chaos, as well as a BCB of a 2-cycle born after a flip bifurcation of the fixed point $R$ (see the arrow marked (2) in Fig. 8a and (3) in Fig. 8b, respectively). In an enlargement of Fig. $8 \mathrm{a}$ shown in Fig. 8b, a bifurcation structure can be seen which has some similarities with a period adding structure; however, an additional study is needed to describe this structure in detail.

In the above examples, the parameter $\rho$, measuring the degree of factor substitutability, plays a crucial role, adding to the complexity of the dynamics, especially when it is negative and it is progressively reduced. However, as it is shown above, due to the discontinuity of the map $F$, complex phenomena, such as, e.g., period adding bifurcation structures with coexistence, emerge also when $\rho$ is negative but not too large in modulus, or even when $\rho$ is positive, i.e., when the degree of factor substitution is quite high.

\subsection{Period adding bifurcation \\ structure in the parameter space of map $g$}

In this section, we describe briefly the period adding structure which can be observed in the feasible parameter domain $\Phi$ of map $F$. As already mentioned, such a structure is associated with transversely attracting cycles belonging to the invariant $x$-axis, on which map $F$ is reduced to a $1 D$ discontinuous map $g$ given in (9).
Recall that a period adding bifurcation structure is formed by infinitely many disjoint periodicity regions, which are ordered according to the Farey summation rule applied to the rotation numbers ${ }^{9}$ of the related cycles. ${ }^{10}$ For example, between the regions related to the cycles with rotation numbers $\frac{1}{n}$ and $\frac{1}{n+1}, n \geq 2$, there exists a region related to the cycles with rotation number $\frac{1}{n} \oplus \frac{1}{n+1}=\frac{2}{2 n+1}$. The construction of a period adding structure starts from the periodicity regions of basic cycles forming two families of the complexity level one: $\left\{L R^{n}, n \geq 1\right\}$ and $\left\{R L^{n}, n \geq 1\right\}$. Then, in the gaps between two consecutive periodicity regions of the complexity level one, the periodicity regions are constructed, associated with four families of the complexity level two: $\left\{L R^{n}\left(R L R^{n}\right)^{m}, m \geq\right.$ $1, n \geq 1\},\left\{\left(L R^{n}\right)^{m} R L R^{n}, m \geq 1, n \geq 1\right\}$, $\left\{R L^{n}\left(L R L^{n}\right)^{m}, m \geq 1, n \geq 1\right\}$ and $\left\{\left(R L^{n}\right)^{m} L R L^{n}\right.$, $m \geq 1, n \geq 1\}$. The related periodicity regions are also disjoint, and in gaps between two consecutive periodicity regions of the complexity level two, the periodicity regions are constructed, related to eight families of the complexity level three. Such a process continues $a d$

\footnotetext{
9 Rotation number of an $n$-cycle of map $g$ with symbolic sequence $\sigma$ can be defined as $\omega(\sigma)=\frac{N_{L}(\sigma)}{n}$, where $N_{L}(\sigma)$ is the number of symbols $L$ in $\sigma$.

${ }^{10}$ More precisely, the Farey summation rule is applied to the rotation numbers $\frac{m_{1}}{n_{1}}$ and $\frac{m_{2}}{n_{2}}$ satisfying $\left|m_{1} n_{2}-m_{2} n_{1}\right|=1$ (such rotation numbers are called Farey neighbors), leading to $\frac{m_{1}}{n_{1}} \oplus \frac{m_{2}}{n_{2}}=\frac{m_{1}+m_{2}}{n_{1}+n_{2}}$.
} 


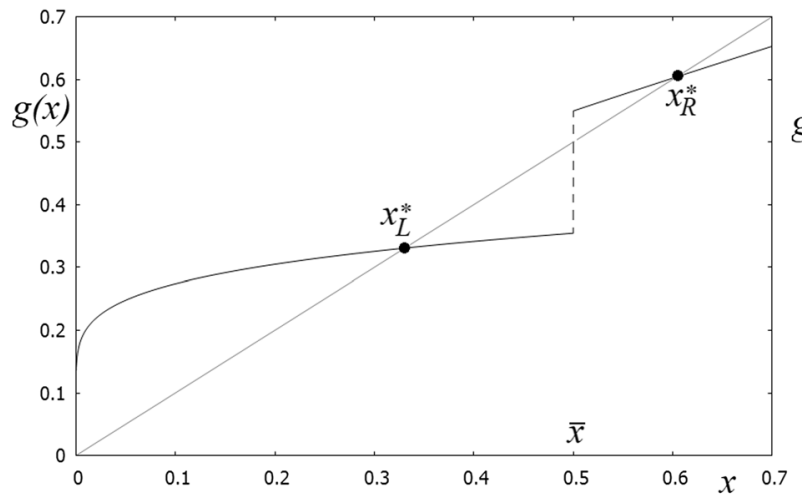

(a)

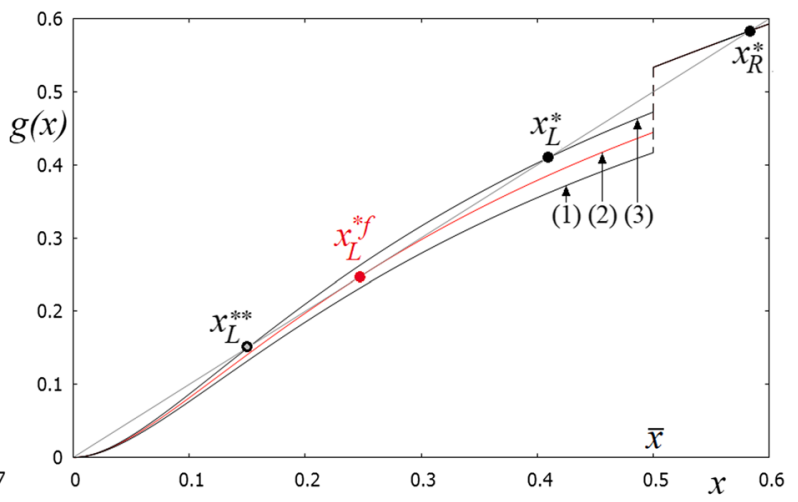

(b)

Fig. 9 Map $g$ for $\mathbf{a} \rho=0.1, w_{1}=0.5$; b $\rho=-1$ and $w_{1}=w_{1}^{f}-0.05$ in (1), $w_{1}=w_{1}^{f}$ in (2), $w_{1}=w_{1}^{f}+0.05$ in (3), where $w_{1}^{f}=0.8$. The other parameters are fixed as $\alpha=0.2, w_{2}=0.4, \bar{x}=0.5$

infinitum, leading to the complete period adding structure (see [4] for details).

It is known that for a $1 \mathrm{D}$ discontinuous map the existence of a period adding structure in the parameter space is associated with increasing branches of the map, or with increasing branches of the proper first return map (see, e.g., [23,29,31-33]). In fact, both functions, $g_{L}$ and $g_{R}$, defining map $g$, are increasing, given that for the considered parameter values and for any $x>0$,

$$
\begin{aligned}
g_{L}^{\prime}(x)= & w_{1}(1-\alpha)(1-\rho) \alpha x^{\rho-1} \\
& \left(1-\alpha+\alpha x^{\rho}\right)^{1 / \rho-2}>0 \\
g_{R}^{\prime}(x)= & w_{2}\left(1+\alpha x^{\rho-1}\right. \\
& \left.\left(1-\alpha+\alpha x^{\rho}\right)^{1 / \rho-1}\right)>0
\end{aligned}
$$

It is also known that for the considered class of maps, an intersection point of two BCB curves related to different attracting fixed points or cycles, can be an issue point of a period adding structure (see, e.g., [23]). The BCB boundaries of the fixed points of map $g$ are given in (26) and (32), respectively, so that the point $P^{*}=B C_{L^{*}} \cap B C_{R^{*}}$ can be defined as

$P^{*}=\left\{\begin{array}{l}w_{1}=\frac{\bar{x}}{(1-\alpha)\left(1-\alpha+\alpha \bar{x}^{\rho}\right)^{1 / \rho-1}} \\ w_{2}=\frac{\bar{x}}{\bar{x}+\left(1-\alpha+\alpha \bar{x}^{\rho}\right)^{1 / \rho}}\end{array}\right.$

Before the investigation of this codimension-two bifurcation point, let us discuss the fixed points of map $g$ in more details.

Consider first map $g_{L}$. It is easy to see that for $\rho<0$ the origin is a fixed point of $g_{L}$ (and this fixed point is superstable: $g_{L}^{\prime}(x)$ can be written as $g_{L}^{\prime}(x)=w_{1}(1-$ $\alpha)(1-\rho) \alpha x^{-\rho}\left((1-\alpha) x^{-\rho}+\alpha\right)^{1 / \rho-2}$, so, $\left.g_{L}^{\prime}(0)=0\right)$, but the origin is not a fixed point for $0<\rho<1$ (in fact, in such a case, $\left.g_{L}(0)=w_{1}(1-\alpha)^{1 / \rho}>0\right)$. From

$$
\begin{aligned}
g_{L}^{\prime \prime}(x)= & w_{1}(1-\alpha)(1-\rho) \alpha x^{\rho-2}\left(1-\alpha+\alpha x^{\rho}\right)^{1 / \rho-3} \\
& \left((\rho-1)(1-\alpha)-\alpha x^{\rho} \rho\right)
\end{aligned}
$$

it follows that if $0<\rho<1$ then $g_{L}^{\prime \prime}(x)<0$ for any $x>0$, that is, $g_{L}$ is concave. Thus, $g_{L}$ can have only one fixed point, denoted $x_{L}^{*}$, which is attracting: using (25), $g_{L}^{\prime}\left(x_{L}^{*}\right)=(1-\rho) \alpha x_{L}^{* \rho}\left(1-\alpha+\alpha x_{L}^{* \rho}\right)^{-1}$, and the inequality $g_{L}^{\prime}\left(x_{L}^{*}\right)<1$, simplifying to $-\rho \alpha x_{L}^{* \rho}<$ $1-\alpha$, is obviously satisfied (see Fig. 9a, where $\rho=0.1$, $\alpha=0.2)$. In the meantime, if $\rho<0$, then at

$x=\left(\frac{(\rho-1)(1-\alpha)}{\alpha \rho}\right)^{1 / \rho}=: \tilde{x}$

the function $g_{L}$ changes its curvature from convex $\left(g_{L}^{\prime \prime}(x)>0\right.$ for $\left.0<x<\tilde{x}\right)$ to concave $\left(g_{L}^{\prime \prime}(x)<0\right.$ for $x>\tilde{x}$ ), that is, $g_{L}$ has an 'S'-shape graph. Clearly, such a graph can have no intersections with the diagonal (except for the origin); it can be tangent to the diagonal at one point (associated with a fold bifurcation), or it can have two intersections with the diagonal (related to two nontrivial fixed points of $\left.g_{L}\right)$. From $g_{L}^{\prime}\left(x_{L}^{*}\right)=1$, we get that at the moment of a fold bifurcation

$x_{L}^{*}=\left(\frac{\alpha-1}{\rho \alpha}\right)^{1 / \rho}=: x_{L}^{* f}$

and this bifurcation occurs if

$F o_{L^{*}}: \quad w_{1}=-\frac{(1-\rho)^{1-1 / \rho}}{\rho \alpha^{1 / \rho}}=: w_{1}^{f}$ 
(note that it is feasible if $\rho<0$ and $w_{1}^{f}<1$ ). So, for $\rho<0$ and $w_{1}<w_{1}^{f}$, the origin is a unique (superstable) fixed point of map $g_{L}$, while for $w_{1}>w_{1}^{f}$ it has two nontrivial fixed points: we keep the notation $x_{L}^{*}$ for an attracting fixed point and denote a repelling fixed point as $x_{L}^{* *}$. Obviously, $x_{L}^{*}>x_{L}^{* *}$, and $x_{L}^{* *}$ separates the basin of the fixed point at the origin from the basin of the fixed point $x_{L}^{*}$.

To give an example, let $\rho=-1$. Then, map $g$ is defined as

$g: x \rightarrow g(x)= \begin{cases}g_{L}(x)=\frac{w_{1}(1-\alpha) x^{2}}{((1-\alpha) x+\alpha)^{2}} & \text { if } x<\bar{x} \\ g_{R}(x)=\frac{\left.w_{2} x(1-\alpha) x+\alpha+1\right)}{(1-\alpha) x+\alpha} & \text { if } x>\bar{x}\end{cases}$

A fold bifurcation occurs at $w_{1}=w_{1}^{f}=4 \alpha$, and for $w_{1}>4 \alpha$ map $g_{L}$ has attracting and repelling fixed points,

$$
\begin{aligned}
x_{L}^{*} & =\frac{w_{1}-2 \alpha+\sqrt{w_{1}^{2}-4 \alpha w_{1}}}{2(1-\alpha)}, \\
x_{L}^{* *} & =\frac{w_{1}-2 \alpha-\sqrt{w_{1}^{2}-4 \alpha w_{1}}}{2(1-\alpha)}
\end{aligned}
$$

respectively. Clearly, both these fixed points are actual for map $g$ only if $x_{L}^{*}<\bar{x}$. We show in Fig. 9b map $g$ at $\rho=-1, \alpha=0.2$ and (1) $w_{1}=w_{1}^{f}-0.05$, that is, before the fold bifurcation; (2) $w_{1}=w_{1}^{f}$, at the moment of the fold bifurcation, with one nontrivial nonhyperbolic fixed point $x_{L}^{* f}=0.25$; (3) $w_{1}=w_{1}^{f}+0.05$, after the fold bifurcation, when two fixed points, $x_{L}^{*}$, and $x_{L}^{* *}$, are born. Here, $w_{1}^{f}=0.8$.

Coming back to the map $g$ given in (9), it is easy to see that the function $g_{R}$ is always concave, given that

$$
\begin{aligned}
g_{R}^{\prime \prime}(x)= & w_{2} \alpha(\rho-1)(1-\alpha) x^{\rho-2} \\
& \left(1-\alpha+\alpha x^{\rho}\right)^{1 / \rho-2}<0
\end{aligned}
$$

For $\rho<0$ the origin is a fixed point of map $g_{R}$ (however, given that $\bar{x}>0$, the origin is never a fixed point of map $g$ ); for $w_{2}<w_{2}^{t r}$, where

$$
w_{2}=\frac{1}{1+\alpha^{1 / \rho}}=: w_{2}^{t r}
$$

the origin is attracting and there are no positive fixed points; at $w_{2}=w_{2}^{t r}$ a transcritical bifurcation occurs, so that for $w_{2}>w_{2}^{t r}$ the origin is a repelling fixed point of $g_{R}$ and there is also a fixed point $x_{R}^{*}>0$ given in (31). For $0<\rho<1$, the origin is not a fixed point of map $g_{R}$ (in fact, $g_{R}(0)=w_{2}(1-\alpha)^{1 / \rho}>0$ ); its unique fixed point $x_{R}^{*}$ (see (31)) satisfies $x_{R}^{*}>0$ if $w_{2}<w_{2}^{t r}$, and $x_{R}^{*} \rightarrow \infty$ as $w_{2} \rightarrow w_{2}^{t r}$. In both cases, for $\rho<0$ or $0<\rho<1$, fixed point $x_{R}^{*}>0$, when it exists, is attracting: using (31), $g_{R}^{\prime}\left(x_{R}^{*}\right)=$ $w_{2}+\alpha x_{R}^{* \rho}\left(1-w_{2}\right)\left(1-\alpha+\alpha x_{R}^{* \rho}\right)^{-1}$, and $g_{R}^{\prime}\left(x_{R}^{*}\right)<1$ if $w_{2}<1$ (see, e.g., the fixed point $x_{R}^{*}$ in Fig. 9).

To summarize, we can state the following

Proposition 2 Map g given in (9) with parameter values satisfying $0<\alpha, w_{1}, w_{2}<1, \rho<1, \rho \neq 0$, $\bar{x}>0$, has

$$
\begin{aligned}
& - \text { an attracting fixed point } x_{R}^{*}=\left(\frac{1-\alpha}{\left(\frac{1}{w_{2}}-1\right)^{\rho}-\alpha}\right)^{1 / \rho} \text { if } \\
& \qquad \alpha<\left(\frac{1}{w_{2}}-1\right)^{\rho} \text { and } x_{R}^{*}>\bar{x} \text {; }
\end{aligned}
$$

- an attracting fixed point at the origin, if $\rho<0$;

- attracting and repelling fixed points, $x_{L}^{*}$ and $x_{L}^{* *}$, respectively, defined implicitly from $x^{\rho}\left((1-\alpha) x^{-\rho}+\right.$ $\alpha)^{1-1 / \rho}=w_{1}(1-\alpha)$, if $\rho<0, w_{1}>-\frac{(1-\rho)^{1-1 / \rho}}{\rho \alpha^{1 / \rho}}$, $x_{L}^{*}<\bar{x}$;

- a repelling fixed point $x_{L}^{* *}$, if $\rho<0, w_{1}>$ $-\frac{(1-\rho)^{1-1 / \rho}}{\rho \alpha^{1 / \rho}}, x_{L}^{*}>\bar{x}, x_{L}^{* *}<\bar{x} ;$

- an attracting fixed point $x_{L}^{*}$ (defined implicitly from $\left.x=w_{1}(1-\alpha)\left(1-\alpha+\alpha x^{\rho}\right)^{1 / \rho-1}\right)$, if $0<\rho<1$, $x_{L}^{*}<\bar{x}$.

Proposition 2 systematizes all the possible stationary equilibria that can exist in a Solow regime. Depending on parameter values, different interesting long-term outcomes may emerge. For example, Fig. 9 shows situations analogous to poverty traps (single or multiple), and Fig. 11 situations similar to boom and bust cycles.

Now we turn to the problem of a period adding structure issuing from point $P^{*}$ (see (43)). To state its existence, we can apply the theorem proved in [23], according to which for map $g$ the inequalities $0<g_{L}^{\prime}\left(x_{L}^{*}\right)<1$ and $0<g_{R}^{\prime}\left(x_{R}^{*}\right)<1$ have to be satisfied for the parameter values corresponding to $P^{*} .^{11}$ Recall that at $P^{*}$ it holds that $x_{L}^{*}=x_{R}^{*}=\bar{x}$ (i.e., map $g$ is continuous), so, we have to check if

$$
\begin{aligned}
\left.g_{L}^{\prime}(\bar{x})\right|_{P^{*}} & =\frac{(1-\rho) \alpha \bar{x}^{\rho}}{\left(1-\alpha+\alpha \bar{x}^{\rho}\right)}<1 \\
\left.g_{R}^{\prime}(\bar{x})\right|_{P^{*}} & =\frac{\bar{x}\left(1+\alpha \bar{x}^{\rho-1}\left(1-\alpha+\alpha \bar{x}^{\rho}\right)^{1 / \rho-1}\right)}{\bar{x}+\left(1-\alpha+\alpha \bar{x}^{\rho}\right)^{1 / \rho}}<1
\end{aligned}
$$

11 It can be checked that other conditions of this theorem are also satisfied, namely, a neighborhood of $P^{*}$ exists, which is divided by the $\mathrm{BCB}$ curves $B C_{L^{*}}$ and $B C_{R^{*}}$ into four subregions: in one of them two attracting fixed points coexist, in another one there are no fixed points (and a period adding structure exists), and in each of two remaining subregions there is just one fixed point. 


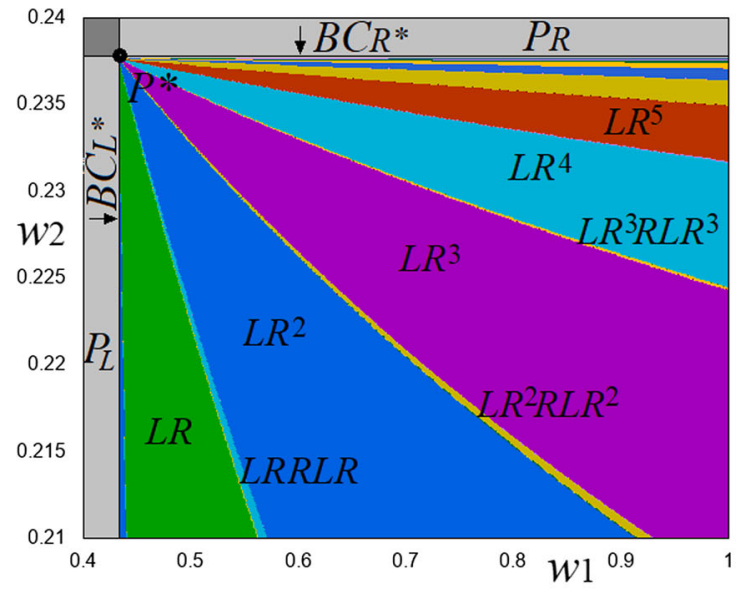

(a)

Fig. 10 Period adding bifurcation structure in the $\left(w_{1}, w_{2}\right)$ parameter plane of map $g$ for $\mathbf{a} \alpha=0.5, \rho=0.5, \bar{x}=0.15$; $\mathbf{b}$ $\alpha=0.2, \rho=-1, \bar{x}=0.5$. In $\mathbf{b}$ the origin is a unique attracting

The second condition, which simplifies to $1-\alpha>0$, is always satisfied; the first condition, which simplifies to $1-\alpha>-\rho \alpha \bar{x}^{\rho}$, is satisfied for $\rho>0$, while for $\rho<0$ this condition can be written as $\alpha<1 /\left(1-\rho \bar{x}^{\rho}\right)$.

In particular, for $\alpha=0.5, \rho=0.5, \bar{x}=0.15$, it holds that $\left.g_{L}^{\prime}(\bar{x})\right|_{P^{*}} \approx 0.14<1,\left.g_{R}^{\prime}(\bar{x})\right|_{P^{*}} \approx$ $0.45<1$, so, point $P^{*}$ defined by $\left(w_{1}, w_{2}\right) \approx$ $(0.4325,0.2377)$, is indeed an issue point of a period adding structure (see Fig. 10a, as well as Fig. 2 which is related to this example). It can also be checked that for $\alpha=0.2, \rho=-1, \bar{x}=0.5$, we have $\left.g_{L}^{\prime}(\bar{x})\right|_{P^{*}}=\frac{2}{3}<$ $1,\left.g_{R}^{\prime}(\bar{x})\right|_{P^{*}} \approx 0.583<1$; thus, point $P^{*}$, defined by $\left(w_{1}, w_{2}\right) \approx(0.9,0.375)$, also gives origin of a period adding structure (see Figs. $10 \mathrm{~b}$ and $4 \mathrm{a}$ ).

In Fig. 10b, the boundary $H_{L^{* *}}$ is shown which is related to a homoclinic bifurcation of the repelling fixed point $x_{L}^{* *}$. This bifurcation destroys an absorbing interval $J=\left[g_{R}(\bar{x}), g_{L}(\bar{x})\right]$, and it occurs if $x_{L}^{* *}=g_{R}(\bar{x})$. The fixed point $x_{L}^{* *}$ remains homoclinic as long as $g_{R}\left(g_{L}(\bar{x})\right)>x_{L}^{* *}$. For $\rho=-1$, using (45), it holds that

$H_{L^{* *}}: \quad w_{2}=\frac{\left(w_{1}-2 \alpha-\sqrt{w_{1}^{2}-4 \alpha w_{1}}\right)((1-\alpha) \bar{x}+\alpha)}{2(1-\alpha) \bar{x}((1-\alpha) \bar{x}+\alpha+1)}$

For the parameter values related to Fig. 10b, the above curve is defined by

$H_{L^{* *}}: w_{2}=\frac{3\left(w_{1}-0.4-\sqrt{w_{1}^{2}-0.8 w_{1}}\right)}{6.4}$

In Fig. 10b, it is also shown the boundary $H_{L^{* *}}^{\prime}$, corresponding to the condition $g_{R}\left(g_{L}(\bar{x})\right)=x_{L}^{* *}$, so that in
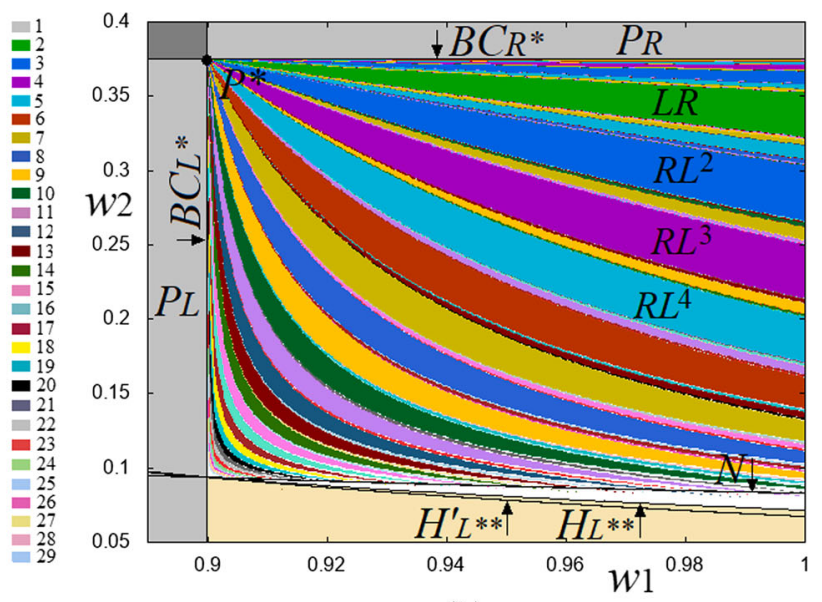

(b)

fixed point of $g$ for the region shown in light yellow (below $H_{L^{* *}}$ and to the right of $B C_{L^{*}}$ ), while in the other part of the parameter plane it coexists with other attractors. (Color figure online)

the narrow region between the curves $H_{L^{* *}}^{\prime}$ and $H_{L^{* *}}$ the fixed point $x_{L}^{* *}$ is homoclinic. ${ }^{12}$ In Fig. $10 \mathrm{~b}$ one more boundary is presented, denoted $N$ and defined by $g_{R} \circ g_{L}(\bar{x})=g_{L} \circ g_{R}(\bar{x})$. It is related to a change of invertibility of map $g$ in the absorbing interval $J$, namely, for the parameter values above $N$ map $g$ is invertible in $J$, being called a gap map, while below $N$ (but above $H_{L^{* *}}$ ) map $g$ is noninvertible in $J$, being called an overlapping map. It is known that, differently from a gap map, an overlapping map can have chaotic attractors, as well as coexisting attractors (see [4] for an overview of the properties of 1D discontinuous piecewise increasing maps).

Figure 11a illustrates the period adding structure, shown in Fig. 10b, by a 1D bifurcation diagram $w_{2}$ versus $x$ for fixed $w_{1}=0.925$, where the borders of the absorbing interval $J=\left[g_{R}(\bar{x}), g_{L}(\bar{x})\right]$ and the repelling fixed point $x=x_{L}^{* *}$, are also presented. Figure $11 \mathrm{~b}$ shows map $g$ and its attracting 15-cycle coexisting with the superstable fixed point at the origin (where $x_{L}^{* *}$ separates their basins) for $w_{2}=0.1$; it holds that $x_{L}^{* *}<g_{R}(\bar{x})$, but by decreasing $w_{2}$ a homoclinic bifurcation of $x_{L}^{* *}$ occurs at $w_{2}=w_{2}^{H} \approx 0.0867$ (when the curve $H_{L^{* *}}$ is crossed, see Fig. 10b), after which a generic trajectory converges to the origin (recall that for map $F$ in the feasible parameter domain $\Phi$ the fixed point $O(0,0)$ is a saddle).

12 This means, in particular, that infinitely many repelling cycles exist, as well as aperiodic trajectories, and map $g$ is chaotic. 


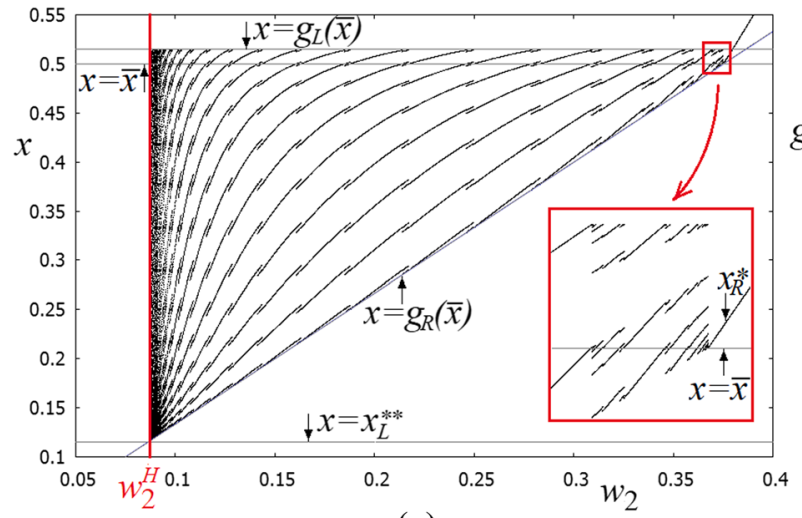

(a)

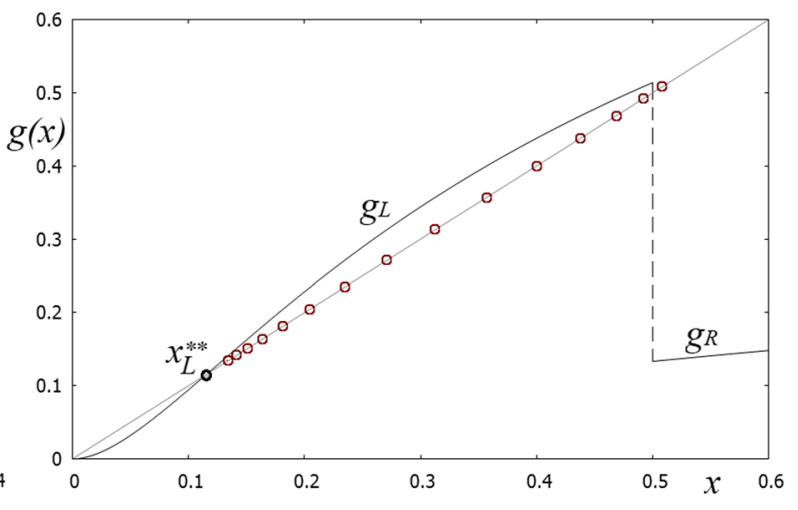

(b)

Fig. 11 a $1 \mathrm{D}$ bifurcation diagram $w_{2}$ versus $x$ of map $g$ for $\alpha=0.2, \rho=-1, \bar{x}=0.5, w_{1}=0.925$; an inset shows the indicated window enlarged; $\mathbf{b}$ an attracting 15-cycle of map $g$ for $w_{2}=0.1$, coexisting with the attracting fixed point at the origin

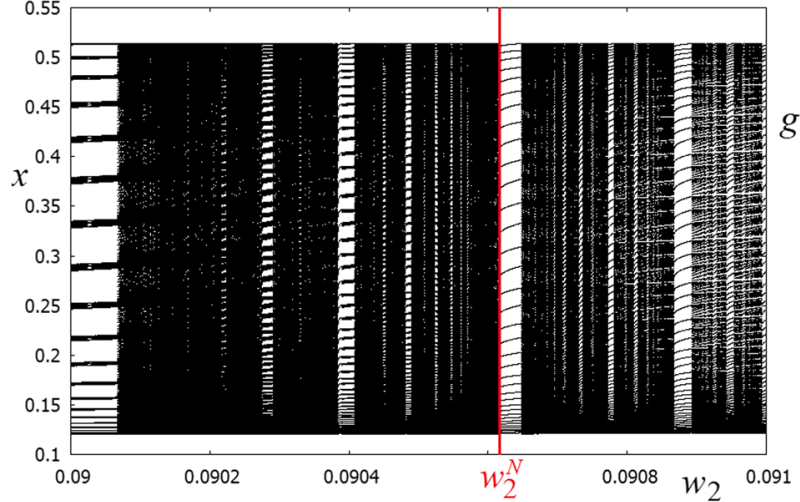

(a)

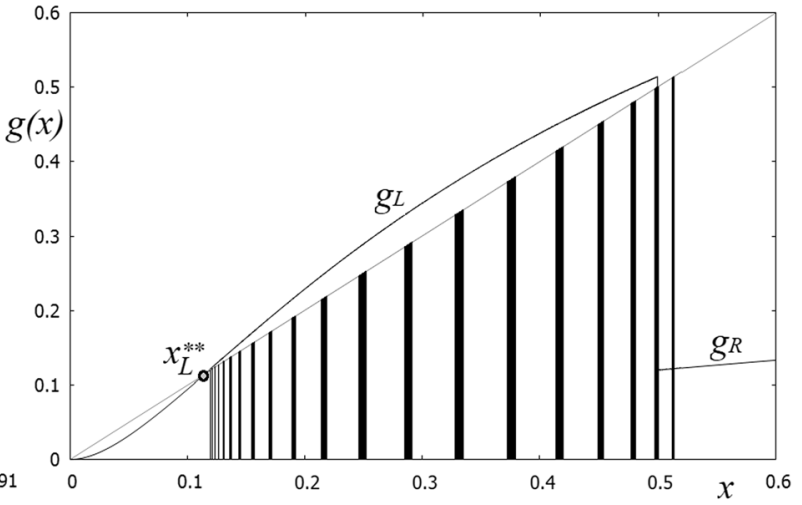

(b)

Fig. 12 a An enlarged part of the 1D bifurcation diagram $w_{2}$ versus $x$ of map $g$ for $\alpha=0.2, \rho=-1, \bar{x}=0.5, w_{1}=0.925$ (see Fig. 11a); b a 20-band chaotic attractor of map $g$ for $w_{2}=0.09$, coexisting with the attracting fixed point at the origin

Figure 12a presents an enlarged part of Fig. 11a, where, in particular, a cross section of the curve $N$ is illustrated, and elements of a bandcount adding bifurcation structure, associated with chaotic attractors, can be recognized. Namely, for $w_{2}^{H}<w_{2}<w_{2}^{N}$, where $w_{2}^{N} \approx 0.0906$, map $g$ is noninvertible on the absorbing interval $J$, and it has chaotic attractors belonging to $J$, as, e.g., a 20-band chaotic attractor shown in Fig. 12b. For detailed description of the bandcount adding bifurcation structure we refer to [4].

As a conclusion about the existence of a period adding structure related to map $g$, we can state the following

Proposition 3 Consider map $g$ given in (9). Let $0<$ $\alpha<1, \rho<1, \rho \neq 0, \bar{x}>0$, be fixed. In the $\left(w_{1}, w_{2}\right)$ parameter plane, the point $P^{*}=B C_{L^{*}} \cap B C_{R^{*}}$ defined in (43) belongs to the feasible domain if

$$
\frac{\bar{x}}{(1-\alpha)\left(1-\alpha+\alpha \bar{x}^{\rho}\right)^{1 / \rho-1}}<1
$$

and it is an issue point of a period adding bifurcation structure if

$\alpha<\frac{1}{1-\rho \bar{x}^{\rho}}$

\subsection{Extension of the period adding structure: a discussion on the set $P$}

In this section, we discuss how the period adding structure associated with map $g$ evolves in the parameter space of map $F$. We are interested in the parameter setting such that set $P$ given in (42) belongs to the feasible domain $\Phi$ defined in (20), and such that set $P$ is related to the BCBs of attracting fixed points. 


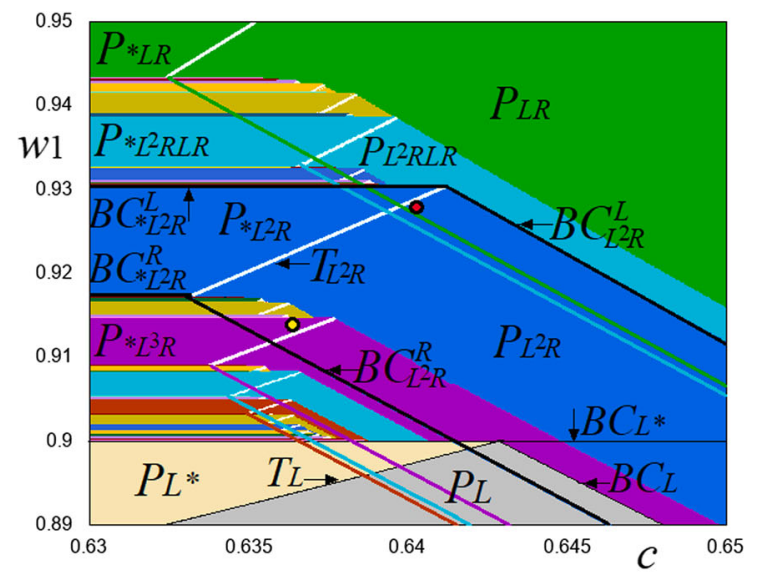

(a)

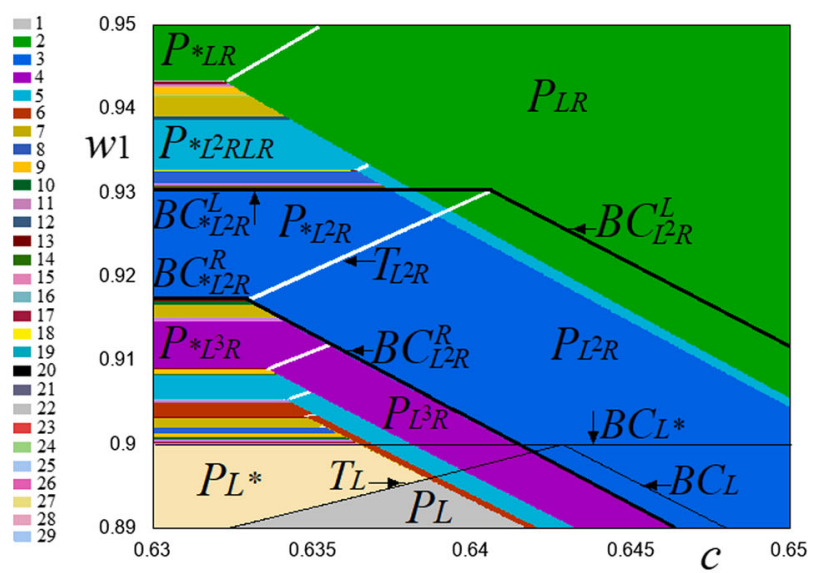

(b)

Fig. 13 Bifurcation structure of the $\left(c, w_{1}\right)$-parameter plane (see window I indicated in Fig. 4a), obtained for different initial points: $x_{0}=0.5, y_{0}=0.0001$ in (a) and $x_{0}=0.51, y_{0}=0.5$ in (b). Other parameters are fixed as $\alpha=0.2, \rho=-1, \bar{x}=0.5, w_{2}=0.35$

As a basic example, let us consider the $\left(c, w_{1}, w_{2}\right)$ parameter space of map $F$ for $\rho=-1, \alpha=0.2$, $\bar{x}=0.5$. We discuss first the bifurcation structure of the enlarged window $\mathrm{I}$ in the $\left(c, w_{1}\right)$-parameter plane, indicated in Fig. 4a. This structure is associated with attracting cycles belonging to the $x$-axis (whose periodicity regions form a period adding structure described in the previous section), which by increasing $c$ lose their transverse stability that leads to the appearance of corresponding interior attracting cycles.

Let $P_{\sigma}$ denote a periodicity region related to an attracting interior cycle with symbolic sequence $\sigma$. In the present section, to distinguish an interior from a border cycle with the same symbolic sequence, we denote a border cycle as $* \sigma$, for example, $L^{2} R$ denotes an interior 3-cycle, while ${ }^{*} L^{2} R$ denotes a border 3-cycle.

Figure 13 shows the bifurcation structure of the window I indicated in Fig. 4a, produced for two different initial conditions, namely, Fig. 13a is related to an initial condition taken close to the $x$-axis, and Fig. $13 \mathrm{~b}$ is produced for an initial condition taken relatively far from the $x$-axis. In Fig. 13, we show also boundaries related to the transverse stability of the border cycles (see the white curves): crossing such a boundary; for example, increasing $c$, the border cycle ${ }^{*} \sigma$ becomes transversely repelling while an interior attracting cycle with the same symbolic sequence $\sigma$ appears in the positive quadrant of the phase plane. Comparing Fig. 13a, $\mathrm{b}$, we can observe overlapping parts of some periodicity regions. In particular, in Fig. 13a, we show by a green line the lower boundary of the region $P_{L R}$ (compare it with the region $P_{L R}$ in Fig. 13b), so that it can be seen that the region $P_{L R}$ overlaps not only with $P_{L^{2} R}$, but also with infinitely many other periodicity regions. These regions are associated with border and interior cycles whose symbolic sequences are located in the Farey tree between $L R$ and $L^{2} R$, and the overlapping mentioned above means that the related cycles coexist. As an example, we show in Fig. 14a coexisting attracting cycles $L R, L^{2} R$ of the first complexity level, as well as cycles $L^{2} R L R,\left(L^{2} R\right)^{2} L R$ of the second complexity level. (The related parameter point is marked by a red circle in Fig. 13a.) One more example is presented in Fig. 14b, where cycles $L^{2} R$, ${ }^{*} L^{3} R$ of the first complexity level and cycles $L^{2} R L^{3} R$, $\left(L^{2} R\right)^{2} L^{3} R, L^{2} R\left(L^{3} R\right)^{2}$ of the second complexity level coexist. (The related parameter point is marked by a yellow circle in Fig. 13a.) Clearly, to get the symbolic sequences of an interior cycle of the second complexity level, the same concatenation rule can be applied as the one associated with the standard period adding bifurcation structure. Note that in both examples presented in Fig. 14, the basins of coexisting attractors are separated by proper segments of the discontinuity line $D L$ and preimages of these segments.

Recall that periodicity regions with horizontal boundaries are related to a period adding structure associated with map $g$; and that crossing an upper (or lower) horizontal boundary of a periodicity region $P_{*} \sigma$ leads to a $\mathrm{BCB}$ of the attracting border cycle ${ }^{*} \sigma$ at which its point, closest to the discontinuity point $x=\bar{x}$ from the left (respectively, right) side, collides with this discontinu- 


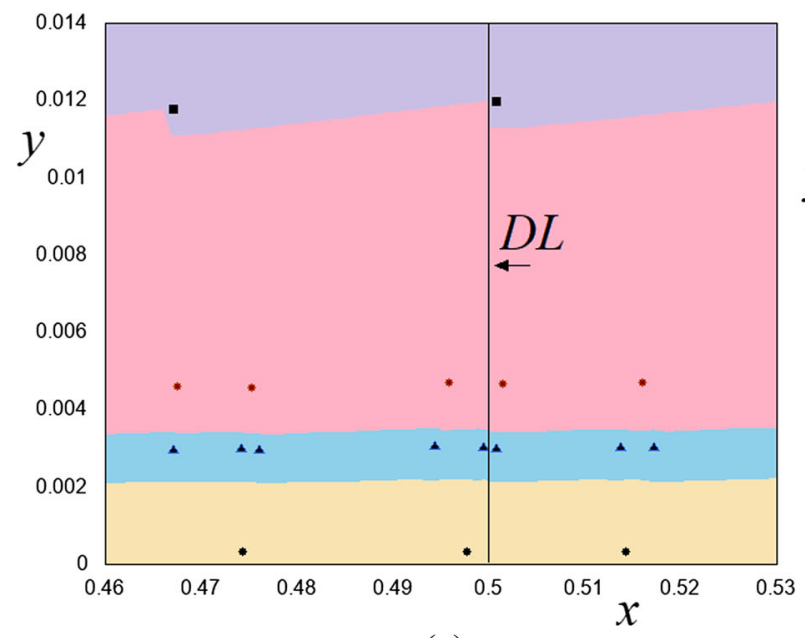

(a)

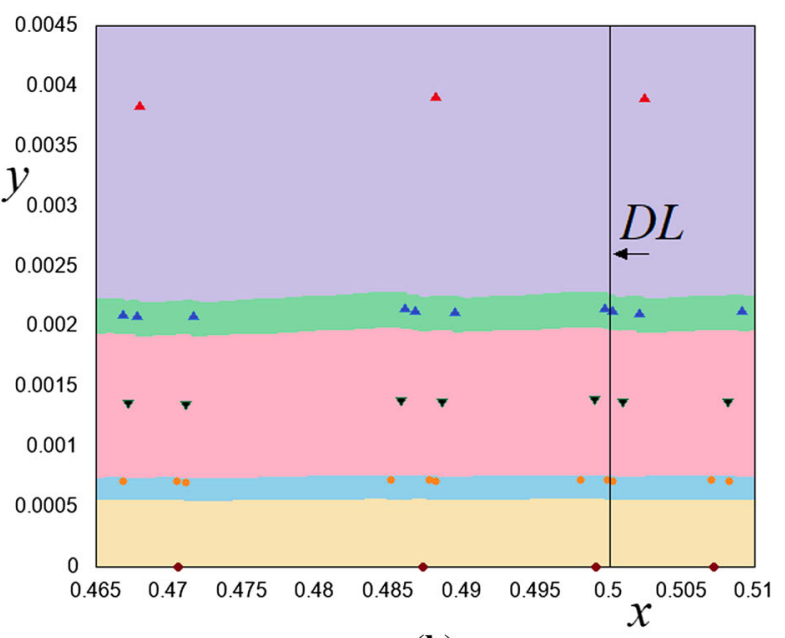

(b)

Fig. 14 Coexisting attracting cycles and their basins for $c=0.64, w_{1}=0.928$ in (a) and $c=0.636534, w_{1}=0.914091$ in (b). Other parameters: $\alpha=0.2, \rho=-1, \bar{x}=0.5, w_{2}=0.35$

ity point. After that, the cycle disappears. It occurs, for example, crossing the boundaries $B C_{* L^{2} R}^{L}$ and $B C_{* L^{2} R}^{R}$ of the region $P_{*} L^{2} R$ indicated in Fig. 13, which are defined by the following conditions:

$$
\begin{aligned}
& B C_{{ }^{*} L^{2} R}^{L}: \bar{x}=g_{L} \circ g_{R} \circ g_{L}(\bar{x}) \\
& B C_{{ }^{*} L^{2} R}^{R}: \bar{x}=g_{L}^{2} \circ g_{R}(\bar{x})
\end{aligned}
$$

The boundaries of $P_{\sigma}$ associated with an interior attracting cycle $\sigma$ are no longer horizontal; however, they correspond to similar bifurcations: crossing an upper (or lower) boundary of such a region leads to a BCB of the interior cycle $\sigma$ at which its point, closest to the discontinuity line $D L$ from the left (respectively, right) side, collides with this discontinuity line and then the interior cycle $\sigma$ disappears. For example, if the BCB boundary $B C_{L^{2} R}^{L}$ or $B C_{L^{2} R}^{R}$, indicated in Fig. 13, is crossed moving from inside to outside the region $P_{L^{2} R}$, the cycle $L^{2} R$ disappears. These boundaries are defined by the following conditions:

$$
\begin{array}{ll}
B C_{L^{2} R}^{L}: & \left(\bar{x}, y_{1}\right)=F_{L} \circ F_{R} \circ F_{L}\left(\bar{x}, y_{1}\right) \\
B C_{L^{2} R}^{R}:\left(\bar{x}, y_{2}\right)=F_{L}^{2} \circ F_{R}\left(\bar{x}, y_{2}\right)
\end{array}
$$

where $\left\{\left(x_{i}, y_{i}\right)\right\}_{i=0}^{2}$ are points of the cycle $L^{2} R$ with $x_{0}<x_{1}<\bar{x}<x_{2}$. Clearly, the BCB boundaries $B C_{\sigma}^{L}$ and $B C_{\sigma}^{R}$ of any other periodicity region $P_{\sigma}$ can be defined in a similar way, taking into account the symbolic sequence $\sigma$ and proper composite functions.

To discuss how the periodicity regions develop in the $\left(c, w_{1}\right)$-parameter plane, we show in Fig. 15a the bifurcation structure of the enlarged window II marked in Fig. 4a. It is clear that point $P$ satisfies the equations of the BCB boundaries $B C_{\sigma}^{L}$ and $B C_{\sigma}^{R}$ of any periodicity region $P_{\sigma}$, given that at point $P$ maps $F_{L}$ and $F_{R}$ have the same fixed point $(x, y)=(\bar{x}, \bar{y})$, where $\bar{y}=\frac{b-\alpha}{1-\alpha}-\bar{x}$. This means that the boundaries $B C_{\sigma}^{L}$ and $B C_{\sigma}^{R}$ intersect at point $P$. However, one cannot state that in a neighborhood of $P$ these boundaries are related to actual cycles, and that there are no other intersection points of these boundaries, that is, we cannot state that the region $P_{\sigma}$ really issues from $P$. In Fig. 15a one can clearly see that $P$ is an issue point for the regions $P_{R^{k} L}$ for $k=\overline{1,7}$, and region $P_{L^{2} R}$ of the basic cycles, as well as region $P_{R L R^{2} L}$ related to the cycle of the second complexity level. These regions have overlapping parts as illustrated in Fig. 15b that presents the phase plane with coexisting cycles $R L, R^{2} L$ and $R L R^{2} L$ for the parameter values indicated in Fig. 15 a by a white circle.

To illustrate the 3D bifurcation structure associated with set $P$, we show in Fig. 16 a few cross sections of the periodicity regions in the $\left(c, w_{1}, w_{2}\right)$-parameter space for $\alpha=0.2, \rho=-1, \bar{x}=0.5$. In this space, set $P$ is a curve issuing from the point $P^{*}$ at which the boundaries $B C_{L}, B C_{R}, B C_{L^{*}}, B C_{R^{*}}$, $T_{L}$ and $T_{R}$ intersect. As obtained before, an intersection of the boundaries $B C_{L^{*}}$ and $B C_{R^{*}}$ is defined by $\left(w_{1}, w_{2}\right)=(0.9,0.375)$ (see Fig. 10b). Using the equation of any other boundary, we get that for the considered parameter values the point $P^{*}$ is determined by $\left(c, w_{1}, w_{2}\right)=(0.64286,0.9,0.375)$. 


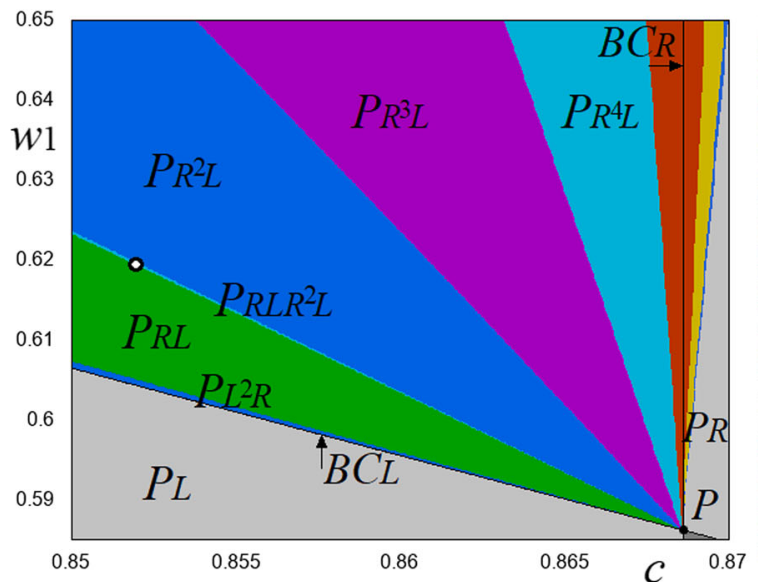

(a)

Fig. 15 a Bifurcation structure of the $\left(c, w_{1}\right)$-parameter plane (an enlargement of window II indicated in Fig. 4a); b coexisting cycles $R L, R^{2} L$ and $R L R^{2} L$ together with their basins for

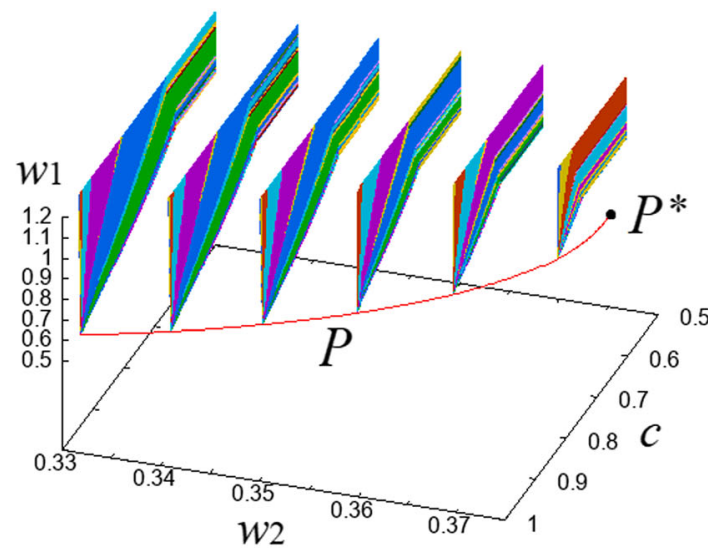

(a)

Fig. 16 Curve $P$ and periodicity regions issuing from this curve in the $\left(c, w_{1}, w_{2}\right)$-parameter space of map $F$, illustrated by a few $\left(c, w_{1}\right)$-cross sections in (a) and $\left(c, w_{2}\right)$-cross sections in

To conclude, we can compare the standard period adding structure associated with the $1 \mathrm{D}$ discontinuous map $g$ and issuing from point $P^{*}$, and a bifurcation structure related to the $2 \mathrm{D}$ discontinuous map $F$ and observed in a neighborhood of set $P$ :

- Both structures are formed by the periodicity regions related to attracting cycles;

- Both structures are associated with an intersection of the BCB boundaries of the stability regions of two different fixed points;

- At point $P^{*}$ it holds that $g_{L}(\bar{x})=g_{R}(\bar{x})=\bar{x}$, that is, map $g$ is continuous, while at set $P$ map $F$

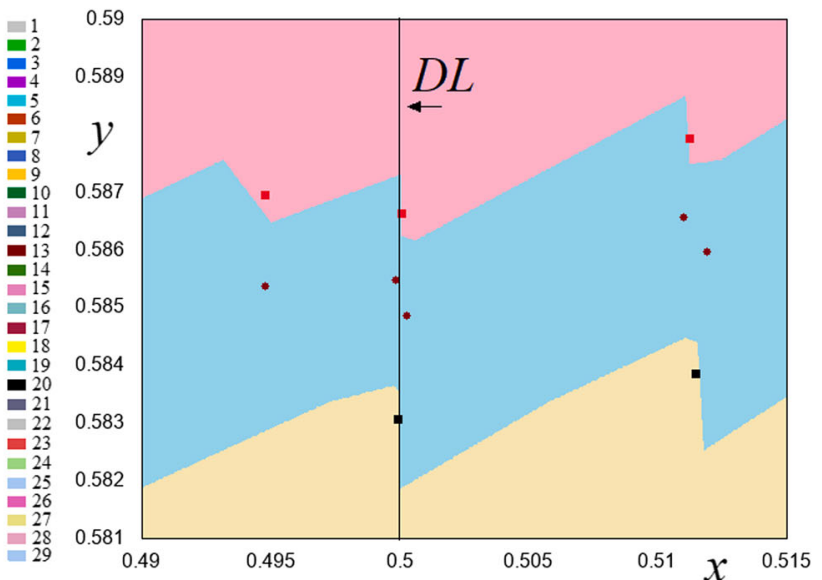

(b)

$c=0.8516, w_{1}=0.6199$ [the related parameter point is marked by white circle in (a)]. Other parameters: $\alpha=0.2, \rho=-1$, $\bar{x}=0.5, w_{2}=0.35$

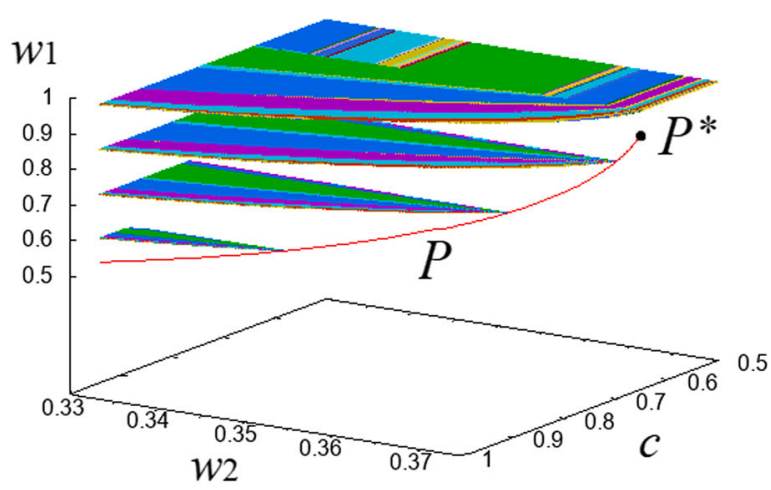

(b)

(b). Here, the stability regions of the fixed points are not shown. Other parameter values are fixed as $\alpha=0.2, \rho=-1, \bar{x}=0.5$

is not continuous: it holds only that the fixed points of both maps, $F_{L}$ and $F_{R}$, undergo a BCB simultaneously: $F_{L}(\bar{x}, \bar{y})=F_{R}(\bar{x}, \bar{y})=(\bar{x}, \bar{y})$;

- All the regions $P_{* \sigma}$ of the period adding structure in the parameter space of map $g$ are disjoint, thus, attracting cycles cannot coexist, differently from the regions $P_{\sigma}$, which can overlap with each other, as well as with stability regions of the fixed points (see, e.g., Fig. 15a where regions $P_{R^{5} L}, P_{R^{6} L}, P_{R^{7} L}$ overlap with region $P_{R}$ ). 


\section{Conclusions}

We considered a two-class economic growth model of the Pasinetti-Solow type. Two heterogeneous groups of agents differentiated according to "social classes," workers and capitalists inhabit the economy. Both may own capital, the only form of wealth in the economy; their saving behavior is socially determined and framed into an overlapping generations structure. Capitalists' utility function always involves an altruistic motive leading to bequests, thus providing for their "dynasty." Workers may switch between two different patterns of saving behavior: when their capital is below a given threshold, they base their saving decision on life-cycle considerations (involving no bequests) - in that case, the old generation of workers consume all their wealth. Instead, when it is above, they start to imitate capitalists and bequest a fraction of their wealth to the young generation. Typically, the dynamic evolution of a twoclass growth model involves two different regimes corresponding to different growth paths. In the Pasinetti regime, both workers and capitalists own a positive amount of capital and the ensuing dynamics is twodimensional; in a Solow regime, only workers own capital and the dynamics is one-dimensional, trapped on the horizontal axis. It is possible to identify the structure of long-term attractors (fixed points, periodic or chaotic) for the two different regimes. However, the switching of workers saving behavior introduces a discontinuity in the corresponding dynamical system and has crucial effects on the properties of the two-class growth model dynamics. Because of the discontinuity, the dynamical system is described by a family of two-dimensional discontinuous piecewise smooth maps. Maps of this class belong to a field in the study of discrete-time dynamical systems which is relatively new and has not been fully explored. Indeed, our model is a source of dynamical phenomena interesting both from a theoretical and applied point of view. For example, we found that the one-dimensional dynamics of the Solow regime, which is able to generate a period adding bifurcation structure, evolves into a peculiar bifurcation structure associated with interior cycles belonging to the Pasinetti regime, when the related attracting cycles lose their transverse stability via transcritical bifurcations. The interesting feature of the observed twodimensional structure is related to multistability, which is impossible in the case of a standard one-dimensional period adding structure. This result is also of interest from an economic point of view revealing the pervasivity of coexistence of different attractors that could belong to one or to both growth regimes and that is present even allowing for strong factor substitutability.

Finally, we confined our study only to some aspects of the dynamics and to some parameter ranges, leaving a more detailed study for future work. Possible extensions from an economic point of view would be: verifying the impact on dynamics of different production technologies; examining more in detail the effects on capital accumulation and wealth distribution of different workers' capital thresholds; and, more generally, exploring how the introduction of behavioral aspects, related to changes in social class behavior, may affect the long-run relationship between economic growth and wealth distribution.

Acknowledgements Open access funding provided by Vienna University of Economics and Business (WU). I. Sushko thanks the University of Urbino for the hospitality experienced during her stay there as a visiting professor.

\section{Compliance with ethical standards}

Conflict of interest The authors declare that they have no conflict of interest.

Open Access This article is licensed under a Creative Commons Attribution 4.0 International License, which permits use, sharing, adaptation, distribution and reproduction in any medium or format, as long as you give appropriate credit to the original author(s) and the source, provide a link to the Creative Commons licence, and indicate if changes were made. The images or other third party material in this article are included in the article's Creative Commons licence, unless indicated otherwise in a credit line to the material. If material is not included in the article's Creative Commons licence and your intended use is not permitted by statutory regulation or exceeds the permitted use, you will need to obtain permission directly from the copyright holder. To view a copy of this licence, visit http://creativecommons.org/licenses/by/4.0/.

\section{References}

1. Agliari, A., Böhm, V., Pecora, N.: Endogenous cycles from income diversity, capital ownership, and differential savings. Chaos Solitons Fract. 130, 109435 (2020)

2. Arrow, K.J.: Aspects of the theory of risk-bearing. Yrjo Jahnsson Lectures (1965)

3. Avrutin, V., Schanz, M., Gardini, L.: Calculation of bifurcation curves by map replacement. Int. J. Bifurc. Chaos 20, 3105-3135 (2010)

4. Avrutin, V., Gardini, L., Sushko, I., Tramontana, F.: Continuous and Discontinuous Piecewise-Smooth One- 
Dimensional Maps: Invariant Sets and Bifurcation Structures. World Scientific, Singapore (2019)

5. Baranzini, M.: A Theory of Wealth Distribution and Accumulation. Oxford University Press, Oxford (1991)

6. Böhm, V., Kaas, L.: Differential savings, factor shares, and endogenous growth cycles. J. Econ. Dyn. Control 24, 965-80 (2000)

7. Brianzoni, S., Mammana, C., Michetti, E.: Complex dynamics in the neoclassical growth model with differential savings and non-constant labor force growth. Stud. Nonlinear Dyn. Econom. 11(3) (2007)

8. Brianzoni, S., Mammana, C., Michetti, E.: Local and global dynamics in a discrete time growth model with nonconcave production function. Discrete Dyn. Nat. Soc. 1-22 (2012b)

9. Brianzoni, S., Mammana, C., Michetti, E.: Global attractor in Solow growth model with differential savings and endogenic labor force growth. AMSE Period. Model. Meas. Control Ser. D 29(2), 19-37 (2008)

10. Brianzoni, S., Mammana, C., Michetti, E.: Nonlinear dynamics in a business cycle model with logistic population growth. Chaos Solitons Fract. 40, 717-30 (2009)

11. Brianzoni, S., Mammana, C., Michetti, E.: Variable elasticity of substitution in a discrete time Solow-Swan growth model with differential saving. Chaos Solitons Fract. 45, 98-108 (2012a)

12. Brianzoni, S., Mammana, C., Michetti, E.: Local and global dynamics in a neoclassical growth model with nonconcave production function and nonconstant population growth rate. SIAM J. Appl. Math. 75(1), 61-74 (2015)

13. Budd, C.J., Piiroinen, P.T.: Corner bifurcations in nonsmoothly forced impact oscillators. Physica D 220(2), 127-145 (2006)

14. Commendatore, P. Complex dynamics in a Pasinetti-Solow model of growth and distribution. Econ. Complex. 3(1), summer 2006, 33-55 (2008)

15. Commendatore, P., Palmisani, C.: The Pasinetti-Solow growth model with optimal saving behaviour: a local bifurcation analysis. In: Skiadas, C.H., Dimotikalis, I., Skiadas, C. (eds.) Topics on Chaotic Systems: Selected Papers from CHAOS 2008 International Conference, pp. 87-95. World Scientific, Singapore (2009)

16. De Nardi, M., Yang, F.: Bequests and heterogeneity in retirement wealth. Eur. Econ. Rev. 72(C), 182-196 (2014)

17. De Nardi, M.: Wealth inequality and intergenerational links. Rev. Econ. Stud. 71(3), 743-768 (2004)

18. di Bernardo, M., Budd, C.J., Champneys, A.R., Kowalczyk, P.: Piecewise-Smooth Dynamical Systems: Theory and Applications, Applied Mathematical Sciences 163. Springer, London (2008)

19. Diamond, P.A.: National debt in a neoclassical growth model. Am. Econ. Rev. 55(5), 1126-1150 (1965)

20. Domar, E.D.: Capital expansion, rate of growth, and employment. Econometrica 14, 137-47 (1946)

21. Dutta, P.S., Routroy, B., Banerjee, S., Alam, S.S.: On the existence of low-period orbits in n-dimensional piecewise linear discontinuous maps. Nonlinear Dyn. 53(4), 369-380 (2008)

22. Foley, D.K., Michl, T.R.: Growth and Distribution. Harward University Press, Cambridge (1999)

23. Gardini, L., Avrutin, V., Sushko, I.: Codimension-2 border collision bifurcations in one-dimensional discontinuous piecewise smooth maps. Int. J. Bifurc. Chaos 24(2), 1450024 (2014)

24. Gardini, L., Sushko, I., Matsuyama, K.: Bifurcation structure in a 2D discontinuous map: explanation of fashion cycles. CHAOS 28, 055917 (2018)

25. Grassetti, F., Mammana, C., Michetti, E.: Substitutability between production factors and growth. An analysis using VES production functions. Chaos Solitons Fract. 113, 53-62 (2018)

26. Grassetti, F., Hunanyan, G., Mammana, C., Michetti, E.: A note on the influence of saving behaviors on economic growth. Metroeconomica 70(3), 442-457 (2019)

27. Gu, E.G.: Bifurcations and chaos for $2 \mathrm{D}$ discontinuous dynamical model of financial markets. Int. J. Bifurc. Chaos 27(12), 1750185 (2017)

28. Harrod, R.F.: An essay in dynamic theory. Econ. J. 49, 14-33 (1939)

29. Homburg, A.J.: Global Aspects of Homoclinic Bifurcations of Vector Fields. Springer, Berlin (1996)

30. Kaldor, N.: Alternative theories of distribution. Rev. Econ. Stud. 23, 83-100 (1955-56)

31. Keener, J.P.: Chaotic behavior in piecewise continuous difference equations. Trans. Am. Math. Soc. 261, 589-604 (1980)

32. Leonov, N.N.: Map of the line onto itself. Radiofisika 3, 942-956 (1959)

33. Lyubimov, D.V., Pikovsky, A.S., Zaks, M.A.: Universal Scenarios of Transitions to Chaos via Homoclinic Bifurcations. Harwood Academic, London (1989)

34. Michl, T.R.: Capitalists, Workers, and Fiscal Policy: A Classical Model of Growth and Distribution. Harvard University Press, Harvard (2009)

35. Mira, C.: Embedding of a Dim1 piecewise continuous and linear leonov map into a Dim2 invertible map. In: Bischi, G.I., Chiarella, C., Sushko, I. (eds.) Global Analysis of Dynamic Models for Economics, Finance and Social Sciences. Springer, Berlin (2013)

36. Mira, C., Gardini, L., Barugola, A., Cathala, J.C.: Chaotic Dynamics in Two-Dimensional NonivertibIe Maps, Nonlinear Sciences, Series A. World Scientific, Singapore (1996)

37. Nusse, H.E., Yorke, J.A.: Border-collision bifurcations including period two to period three for piecewise smooth systems. Physica D 57, 39-57 (1992)

38. Nusse, H.E., Yorke, J.A.: Border-collision bifurcations for piecewise smooth one-dimensional maps. Int. J. Bifurc. Chaos 5, 189-207 (1995)

39. Pasinetti, L.: Rate of profit and income distribution in relation to rate of economic growth. Rev. Econ. Stud. 34(4), 267-279 (1962)

40. Rakshit, B., Apratim, M., Banerjee, S.: Bifurcation phenomena in two-dimensional piecewise smooth discontinuous maps. CHAOS 20, 033101 (2010)

41. Ramsey, F.P.: A mathematical theory of saving. Econ. J. 38(152), 543-559 (1928)

42. Rulkov, N.F.: Modeling of spiking-bursting neural behavior using two-dimensional map. Phys. Rev. E 65(4), 041922 (2002)

43. Samuelson, P.A., Modigliani, F.: The Pasinetti Paradox in neoclassical and more general models. Rev. Econ. Stud. 33, 269-301 (1966) 
44. Solow, R.M.: A contribution to the theory of economic growth. Q. J. Econ. 70, 65-94 (1956)

45. Sushko, I., Avrutin, V., Gardini, L.: Bifurcation structure in the skew tent map and its application as a border collision normal form. J. Differ. Equ. Appl. 22(8), 1040-1087 (2015)

46. Tramontana, F., Sushko, I., Avrutin, V.: Period adding structure in a 2D discontinuous model of economic growth. Appl. Math. Comput 253, 262273 (2015)
Publisher's Note Springer Nature remains neutral with regard to jurisdictional claims in published maps and institutional affiliations. 